\title{
Epoxyeicosanoids stimulate multiorgan metastasis and tumor dormancy escape in mice
}

\author{
Dipak Panigrahy,,$^{1,2}$ Matthew L. Edin, ${ }^{3}$ Craig R. Lee, ${ }^{3,4}$ Sui Huang, ${ }^{5,6}$ Diane R. Bielenberg, ${ }^{1}$ \\ Catherine E. Butterfield, ${ }^{1}$ Carmen M. Barnés, ${ }^{1}$ Akiko Mammoto, ${ }^{1}$ Tadanori Mammoto, ${ }^{1}$ Ayala Luria, ${ }^{7}$ \\ Ofra Benny, ${ }^{1}$ Deviney M. Chaponis, ${ }^{1,2}$ Andrew C. Dudley, ${ }^{1}$ Emily R. Greene,, ${ }^{1,2}$ Jo-Anne Vergilio, ${ }^{8}$ \\ Giorgio Pietramaggiori, 9 Sandra S. Scherer-Pietramaggiori, ${ }^{9}$ Sarah M. Short, ${ }^{1}$ Meetu Seth, ${ }^{1}$ \\ Fred B. Lih, ${ }^{3}$ Kenneth B. Tomer, ${ }^{3}$ Jun Yang, ${ }^{7}$ Reto A. Schwendener, ${ }^{10}$ Bruce D. Hammock, ${ }^{7}$ \\ John R. Falck, ${ }^{11}$ Vijaya L. Manthati, ${ }^{11}$ Donald E. Ingber, ${ }^{1,12}$ Arja Kaipainen,13 \\ Patricia A. D'Amore, ${ }^{14}$ Mark W. Kieran, ${ }^{1,2}$ and Darryl C. Zeldin ${ }^{3}$ \\ 1Vascular Biology Program, Children's Hospital Boston, and 2Division of Pediatric Oncology, Dana-Farber Cancer Institute, Harvard Medical School, \\ Boston, Massachusetts, USA. ${ }^{3}$ Division of Intramural Research, National Institute of Environmental Health Science (NIEHS), NIH, Research Triangle Park, \\ North Carolina, USA. ${ }^{4}$ Division of Pharmacotherapy and Experimental Therapeutics, Eshelman School of Pharmacy, University of North Carolina, \\ Chapel Hill, North Carolina, USA. ${ }^{5}$ Institute for Biocomplexity and Informatics, University of Calgary, Calgary, Alberta, Canada. ${ }^{6}$ Institute for Systems Biology, \\ Seattle, Washington, USA. 'Department of Entomology and Cancer Center, UCD, Davis, California, USA. ${ }^{8}$ Department of Pathology, Children's Hospital Boston, \\ Harvard Medical School, Boston, Massachusetts, USA. 9Division of Plastic Surgery, University of Lausanne, Lausanne, Switzerland. \\ ${ }^{10}$ Institute of Molecular Cancer Research, University of Zurich, Zurich, Switzerland. ${ }^{11}$ Department of Biochemistry, \\ University of Texas Southwestern Medical Center, Dallas, Texas, USA. ${ }^{12}$ Wyss Institute for Biologically Inspired Engineering at Harvard University, \\ Boston, Massachusetts, USA. ${ }^{13}$ Department of Biochemistry and Molecular Biology, University of Calgary, Calgary, Alberta, Canada. \\ ${ }^{14}$ Schepens Eye Research Institute, Harvard Medical School, Boston, Massachusetts, USA.
}

\begin{abstract}
Epoxyeicosatrienoic acids (EETs) are small molecules produced by cytochrome $\mathbf{P 4 5 0}$ epoxygenases. They are lipid mediators that act as autocrine or paracrine factors to regulate inflammation and vascular tone. As a result, drugs that raise EET levels are in clinical trials for the treatment of hypertension and many other diseases. However, despite their pleiotropic effects on cells, little is known about the role of these epoxyeicosanoids in cancer. Here, using genetic and pharmacological manipulation of endogenous EET levels, we demonstrate that EETs are critical for primary tumor growth and metastasis in a variety of mouse models of cancer. Remarkably, we found that EETs stimulated extensive multiorgan metastasis and escape from tumor dormancy in several tumor models. This systemic metastasis was not caused by excessive primary tumor growth but depended on endothelium-derived EETs at the site of metastasis. Administration of synthetic EETs recapitulated these results, while EET antagonists suppressed tumor growth and metastasis, demonstrating in vivo that pharmacological modulation of EETs can affect cancer growth. Furthermore, inhibitors of soluble epoxide hydrolase (sEH), the enzyme that metabolizes EETs, elevated endogenous EET levels and promoted primary tumor growth and metastasis. Thus, our data indicate a central role for EETs in tumorigenesis, offering a mechanistic link between lipid signaling and cancer and emphasizing the critical importance of considering possible effects of EET-modulating drugs on cancer.
\end{abstract}

\section{Introduction}

Endogenously produced lipid autacoids are locally acting small molecule mediators that play a central role in inflammation and in the response to tissue injury. Although these autacoids, including prostaglandins and leukotrienes, are best known as products of arachidonic acid metabolism by cyclooxygenases and lipoxygenases (1), arachidonic acid is also a substrate for the cytochrome P450 (CYP) epoxygenases CYP2C8 and CYP2J2, which convert it to 4 regioisomeric EETs (5,6-EET, 8,9-EET, 11,12-EET, and 14,15EET). The bioactive EETs are produced predominantly in the endothelium and are metabolized by soluble epoxide hydrolase (sEH) to less-active dihydroxyeicosatrienoic acids (DHETs) $(2,3)$ (see scheme in Supplemental Figure 1A; supplemental material

Authorship note: Dipak Panigrahy, Matthew L. Edin, Craig R. Lee, and Sui Huang contributed equally to this work.

Conflict of interest: The authors have declared that no conflict of interest exists. Citation for this article: J Clin Invest. 2012;122(1):178-191. doi:10.1172/JCI58128. available online with this article; doi:10.1172/JCI58128DS1). EETs have been investigated as autocrine and paracrine mediators of arachidonic acid-induced vasorelaxation in the cardiovascular and renal systems. Thus, inhibitors of sEH, which raise endogenous EET levels, are in clinical trials as anti-hypertensive agents and are being considered for long-term use in diabetes, stroke, cerebral ischemia, dyslipidemia, pain, immunological disorders, eye diseases, neurological diseases, renal disease, organ damage, vascular remodeling, atherosclerosis, ischemia-reperfusion, lung disease (chronic obstructive pulmonary disease [COPD]), graft stenosis, and other medical conditions $(4,5)$.

Despite their known proangiogenic effects (6-8), the role of EETs in cancer is poorly understood (9). CYP epoxygenases are regulated in human carcinoma tissues and tumor cell lines (10), and this has been associated with increased metastasis (11). Moreover, genetic polymorphisms in CYP2C8 and CYP2C9 influence disease-free survival of breast cancer patients (12). Thus, while existing studies associate CYP enzymes with cancer $(10,11,13)$, the direct role of 
their products, the EETs, in cancer remains unknown. Since EET levels are directly influenced by nutrients and inflammatory processes (14), we investigated the effect of endothelium-derived and systemic EETs on cancer in a variety of animal models. We demonstrate here that elevated EETs have a potent stimulatory effect on primary tumor growth and tumor angiogenesis. Surprisingly, elevated EETs triggered massive, unprecedented patterns of metastatic spread and escape from tumor dormancy.

\section{Results}

Endothelium-derived EETs stimulate primary tumor growth. To determine whether EET autacoids produced by the endothelium promote cancer, we generated three lines of transgenic mice with high endothelial EET levels: mice with endothelial (Tie2 promoterdriven) expression of either human CYP2C8 or human CYP2J2 (Tie2-CYP2C8-Tr, Tie2-CYP2J2-Tr) (15) and mice with global disruption of the gene that encodes $\mathrm{SEH}$ (sEH-null) (16). ECs isolated from Tie2-CYP2C8-Tr and Tie2-CYP2J2-Tr mice had significantly increased EET levels compared with ECs isolated from WT mice as measured by liquid chromatography-tandem mass spectrometry (LC/MS/MS) (Supplemental Figure 1B), and sEH-null mice have significantly increased plasma EETs compared with WT mice (17). In contrast, cyclooxygenase and lipoxygenase metabolites of arachidonic acid are unaffected in these mice. Conversely, to characterize the impact of reduced endothelial EET levels, we also used a novel murine model with endothelial expression of human sEH (Tie2-sEH-Tr) (18). ECs isolated from these mice had significantly decreased EET levels relative to ECs isolated from WT mice (Supplemental Figure 1B).

We first investigated whether EETs could stimulate primary tumor growth in a series of standard syngeneic tumor models using the genetically altered mice that exhibit high endothelial EET levels. We observed a dramatic increase in the growth of B16F10 melanoma, T241 fibrosarcoma, and Lewis lung carcinoma (LLC) in Tie2-CYP2C8-Tr, Tie2-CYP2J2-Tr, and sEH-null mice compared with WT mice, suggesting that endothelial EETs promote primary tumor growth (Figure 1A and Supplemental Figure 1C). Conversely, in Tie2-sEH-Tr mice, there was a $60 \%$ reduction in B16F10 melanoma and T241 fibrosarcoma growth compared with WT mice, suggesting that tumor growth was suppressed in mice with low endothelial EET levels (Figure 1B).

Plasma 11,12-EET and 14-15-EET levels were elevated 15-fold in sEH-null tumor-bearing mice when measured on day 22 after injection of T241 fibrosarcoma (Supplemental Figure 1D). In Tie2CYP2C8-Tr mice, where enhanced EET biosynthesis was designed to be endothelium specific, plasma 14,15 -EET increased by $33 \%$ on day 16 after injection of LLC (Supplemental Figure 1D). The changes in eicosanoid levels were selective for epoxyeicosanoids in that other eicosanoids and related metabolites (19) including $\mathrm{PGE}_{2}$, 6-keto $\mathrm{PGF}_{1 \alpha}$ (stable $\mathrm{PGI}_{2}$ metabolite), $\mathrm{PGD}_{2}$, and several hydroxyeicosatetraenoic acid (HETE) regioisomers were not significantly altered in these models (data not shown). LC/MS/MS analysis of T241 fibrosarcoma tumors from Tie2-CYP2C8-Tr, sEHnull, and WT mice showed no changes in levels of EETs, including 14,15-EET (Supplemental Figure 1E). Since both plasma EET levels and primary tumor growth were increased in genetically altered mice, we reasoned that exogenously administrated EETs might also promote primary tumor growth in WT mice. Indeed, systemic administration of 14,15-EET by osmotic minipump significantly accelerated primary LLC tumor growth (Figure 1C).
To exclude the possibility that the tumor-stimulating effect of EETs was specific to the growth of subcutaneous tumors, we used an orthotopic human prostate cancer model (20). Systemic administration of 14,15 -EET for 28 days to mice injected with human prostate cancer cells (PC3M-LN4) at an inoculation of 10 -fold fewer cells than normally used (20) stimulated orthotopic tumor growth (Supplemental Figure 1E). To further exclude that EETs promoted growth of these tumors via a specific beneficial effect on transplanted tumor cells, we next used an endogenous tumor model. Transgenic adenocarcinoma of the mouse prostate (TRAMP) mice are one of the few genetically engineered murine models of a spontaneous tumor that exhibits widespread metastasis and closely mimics the histological progression of human prostate cancer (21). Plasma from TRAMP mice exhibited higher epoxygenase activity (sum of 14,15-EET and 14,15-DHET), while prostate tumors from TRAMP mice showed no change in 14,15-EET levels relative to normal prostates from age-matched control mice (Supplemental Figure 1, G and H). Control mice at 18 weeks of age (treated with vehicle for 13 weeks) had normalappearing prostates with intraepithelial neoplasia, while mice treated with 14,15-EET for 13 weeks showed large prostate tumors (Figure 1D). In summary, EETs promote tumor growth independent of the specific tumor model used, ranging from subcutaneous and orthotopic transplantable to endogenous tumors.

EETs promote exit from tumor dormancy. Given that EETs stimulated both endogenous and implanted tumors, we asked whether EETs quantitatively increase the growth rate of established tumors and/or accelerate the qualitative transition from a microscopic dormant tumor to a macroscopic growing tumor. This process is often termed escape from dormancy and is thought to be regulated, in part, by the angiogenic switch as well as production of a critical mass of tumor-initiating cells $(22,23)$. Tumor dormancy is believed to be maintained by the suppression of angiogenesis $(24,25)$, while the triggers that initiate growth and angiogenesis of dormant tumor cells are poorly understood. We administered 14,15-EET to mice injected with human liposarcoma cells derived from a clone previously shown to enter a stable period of dormancy (26). Here we observed that systemic administration of 14,15-EET for 113 days to mice injected with 10-fold fewer liposarcoma cells than normally required (26) led to macroscopic tumor growth by day 90 after tumor cell implantation (Figure 1E). In contrast, control animals, receiving vehicle-treated osmotic minipumps, did not exhibit visible tumor growth up to day 190. Thus, EETs accelerate the escape from tumor dormancy.

To further examine the extent to which EETs trigger exit from dormancy, we serially injected decreasing numbers of LLC tumor cells, since implantation below a critical number of tumor cells mimics dormancy $(23,27)$. Systemic administration of 14,15 EET increased the LLC tumor growth rate when a large number $\left(10^{5}, 10^{6}\right)$ of LLC cells was injected; this was achieved without accelerating the time to tumor "take-off." Remarkably, at a lower LLC cell number $\left(10^{4}\right), 14,15$-EET drastically accelerated the time, by 10 days, to tumor take-off, after which the growth rate was similar to that of control LLC tumors (Figure 1C). At $10^{3}$ injected LLC cells, 14,15-EET-treated mice showed macroscopic tumors, albeit with a 32-day delay, growing at the same rate as control mice receiving 10 times more $\left(10^{4}\right)$ cells. By contrast, in control mice injected with $10^{3}$ LLC tumor cells, tumors were not visible and remained dormant during the entire 209-day period of observation (Figure 1C). Collectively, these data demonstrate 

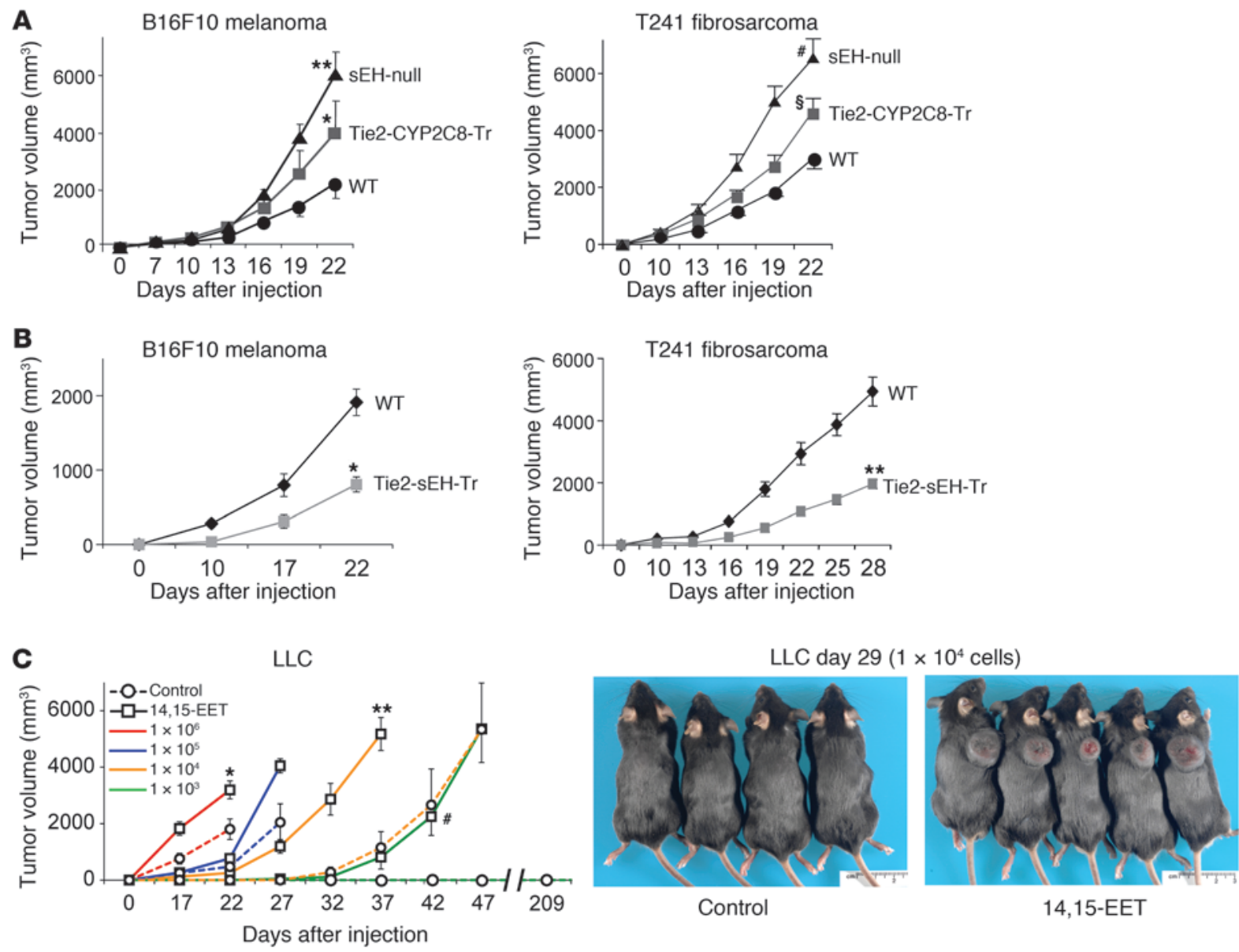

D
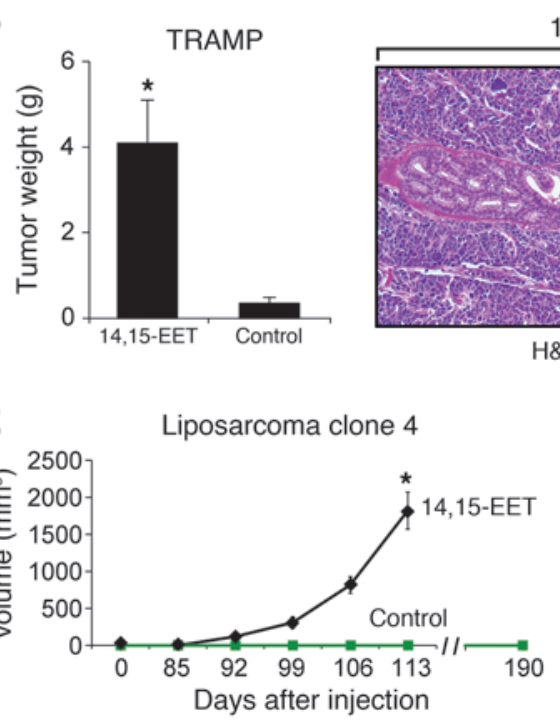

14,15-EET (3.9 g)

Control $(<0.1 \mathrm{~g})$
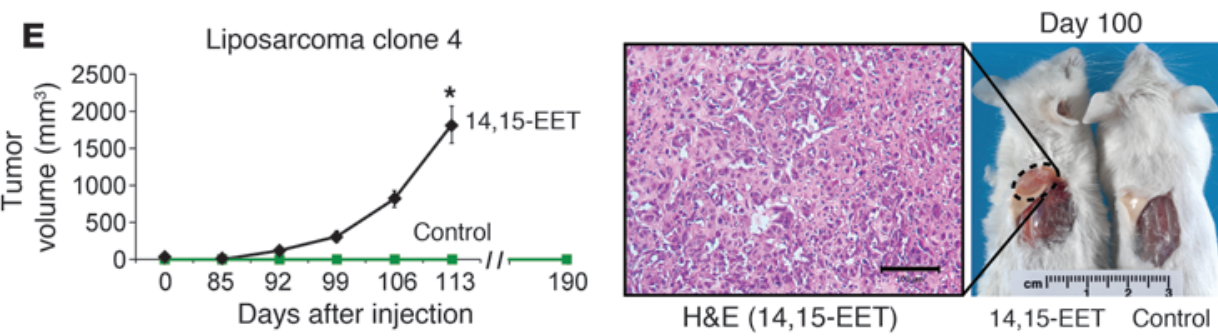


\section{Figure 1}

EETs stimulate primary tumor growth and escape from tumor dormancy. (A) Growth of B16F10 melanoma and T241 fibrosarcoma primary tumors in sEH-null, Tie2-CYP2C8-Tr, and WT mice. $n=10-14$ mice/ group; ${ }^{\star} P=0.042$, ${ }^{* \star} P=0.024,{ }^{\#} P=0.008,{ }^{\S} P<0.001$ versus $\mathrm{WT}$; unpaired Student's $t$ test is used throughout. (B) Primary B16F10 melanoma and T241 fibrosarcoma tumor growth is inhibited in Tie2-sEHTr mice on days 22-28. $n=5-6$ mice/group; ${ }^{*} P=0.001$, ${ }^{* *} P=0.006$ versus WT. (C) Systemic administration of $14,15-$ EET $(15 \mu \mathrm{g} / \mathrm{kg} / \mathrm{d})$ via osmotic minipump stimulates primary LLC tumor growth. When $1 \times 10^{4}$ or $1 \times 10^{3}$ LLC cells were injected, 5 of 9 control mice and 9 of 9 control mice, respectively, did not exhibit visible tumor growth up to 209 days after injection. Images show representative mice on day 29 after injection with $1 \times 10^{4}$ LLC cells. $n=6-9$ mice/group; ${ }^{*} P \leq 0.02,{ }^{*} P \leq 0.001$, ${ }^{\#} P \leq 0.006$ versus WT. The experiment was performed 3 times with similar results. Rulers show centimeters. (D) 14,15-EET (15 $\mu \mathrm{g} / \mathrm{kg} / \mathrm{d})$ stimulates tumor growth in a genetically engineered model of cancer (TRAMP). Ruler shows centimeters. At 90 days of treatment, H\&Estained section of prostate of control TRAMP mice reveals prostatic intraepithelial neoplasia (PIN) surrounded by tumor cells, while H\&Estained section of prostate of TRAMP mice treated with 14,15-EET shows glandular parenchyma surrounded by tumor cells. $n=6$ mice/ group; ${ }^{*} P=0.002$ versus control. Scale bars: $100 \mu \mathrm{m}$. (E) 14,15-EET accelerates escape in a tumor dormancy model of human liposarcoma cells (clone 4). After treatment with 14,15-EET for 100 days, large palpable liposarcoma tumors are visible. No tumor is visible in the control mice. Ruler shows centimeters. H\&E-stained section of tumors of mice treated with 14,15-EET reveal sheets of lipoblasts. $n=5-6$ mice/group; ${ }^{*} P=0.008$ versus control. Scale bar: $100 \mu \mathrm{m}$.

that below a critical threshold of tumor cell number, EETs are necessary for normal tumor development.

EETs enhance tumor angiogenesis. To determine whether tumor angiogenesis contributed to the increase in escape rate as well as primary tumor growth rate in the genetically altered and 14,15-EETtreated mice, we analyzed the amount of endothelium in tumors by immunohistochemical detection of the EC marker CD31 and by flow cytometry. Immunohistochemistry studies showed an increase in CD31-positive vessels in B16F10 tumors on day 22 after injection in Tie2-CYP2C8-Tr, Tie2-CYP2J2-Tr, and sEH-null mice relative to WT mice (Supplemental Figure 1I). Likewise, flow cytometry of dissociated tumor cells revealed a significant increase in CD31positive ECs in LLC tumors from Tie2-CYP2J2-Tr mice compared with WT mice on day 22 after injection (Supplemental Figure 1J). Moreover, tumor-associated angiogenesis increased in 14,15-EETtreated prostate (PC3M-LN4 and TRAMP) tumors (Supplemental Figure 1K). Conversely, CD31-positive vessels decreased in T241 fibrosarcoma tumors in Tie2-sEH-Tr mice relative to WT mice on day 28 after tumor implantation (Supplemental Figure 1L).

To determine whether increased angiogenesis in tumors was a consequence of faster tumor growth or a primary response to EETs, we performed a corneal tumor angiogenesis assay. This is a standard assay in which LLC is implanted into a cornea micropocket and allowed to stimulate neovascularization (28). We observed dramatic stimulation of tumor-dependent angiogenesis in Tie2-CYP2C8-Tr and sEH-null mice on day 13 after LLC implantation in the cornea (Supplemental Figure 1M). Likewise, systemic administration of 14,15-EET enhanced corneal tumor angiogenesis when LLC-GFP and B16F10 melanoma were implanted in the cornea, as reflected by a $23 \%$ increase in CD31-positive ECs (Supplemental Figure 1, N and O). These cornea assays allowed us to monitor angiogenesis before tumor mass development.
Administration of synthetic EETs or genetic disruption of sEH, the EETs' primary degrading enzyme, increased systemic EET levels and mimicked the effects of endothelium-derived EETs in the Tie 2 promoter-driven CYP transgenic mice. However, we have not excluded the possibility that in the latter animals, EETs produced by monocytes/macrophages could account for the tumorpromoting effects of EETs, since the Tie 2 promoter is also active in monocytes/macrophages (29). We therefore depleted macrophages with clodronate liposomes (30) in Tie2-CYP2C8-Tr- and 14,15-EET-treated mice (Supplemental Figure 1P) and observed no effect on LLC tumor growth.

EETs stimulate multiorgan metastasis. By manipulating EET levels in the tissue, as opposed to CYP expression in tumor cells (as previously reported; ref. 11), we can now study the direct, systemic role of EETs in cancer metastasis. Using a well-established model in which resection of a primary tumor reproducibly stimulates development of distant metastasis 14-17 days after resection $(31,32)$, we investigated whether EETs promote spontaneous metastatic growth (as opposed to inducing metastatic tumors by intravenous injection of tumor cells). Autopsies of moribund mice 10 days after resection of primary LLC revealed a dramatic increase in lung weight and in surface lung metastases in both Tie2-CYP2C8-Tr and sEH-null mice compared with WT mice (Figure $2 \mathrm{~A}$ ). In sEH-null mice, the normal lung tissue was completely replaced by invasive metastatic lesions (Figure 2A). Tie2-CYP2C8$\mathrm{Tr}$ and sEH-null mice also exhibited spontaneous liver and kidney metastasis (Supplemental Figure 2A). In contrast, there was a 50\% decrease in the number of lung metastases at day 17 in Tie2-sEHTr mice when compared with WT mice (Figure 2B and Supplemental Figure 2B). These observations indicate that EETs stimulate spontaneous metastasis following resection of a primary tumor and, more strikingly, that they are important for the normally observed basal metastatic rate in WT mice.

Surprisingly, even without resection of the primary tumor, LLC metastases were observed in axillary lymph nodes and lungs of $100 \%$ of the Tie2-CYP2J2-Tr mice by day 22 after injection (Supplemental Figure 2, C and D). In contrast, no gross metastases were observed in Tie2-CYP2J2-Tr mice injected with T241 fibrosarcoma.

To determine whether the pro-metastatic effect of endotheliumderived EETs was limited to the LLC model, we injected B16F10 melanoma cells via the tail vein into Tie2-CYP2C8-Tr mice. In this common (non-spontaneous) hematogenic metastasis model, B16F10 cells exclusively colonize the lung and produce pulmonary metastases (33). However, in the Tie2-CYP2C8-Tr mice, B16F10 melanoma cells produced macroscopic metastasis not only in lung but also in liver (Supplemental Figure 2E). This is of interest because liver metastasis is commonly only achieved by direct injection of B16F10 melanoma cells into the portal or splenic vein (34). In parallel experiments, when primary subcutaneous $\mathrm{B} 16 \mathrm{~F} 10$ tumors were resected to trigger distant metastasis (as in the LLC model), there was a large (3-fold) increase in axillary lymph node metastasis in both Tie2-CYP2C8-Tr and sEH-null mice 17 days after B16F10 melanoma resection (Supplemental Figure 2F). This suggests that macrometastatic lesions stimulated by EETs are independent of tumor type. Importantly, systemic administration of 14,15-EET via osmotic minipumps in WT mice at the time of LLC tumor resection also stimulated a 3-fold increase in the number of surface lung metastases compared with vehicle-treated controls and led to an induction of liver, kidney, and distant lymph node metastasis 12 days after resection of the primary LLC tumor (Figure 2C and Supplemental Figure 2G). 
A

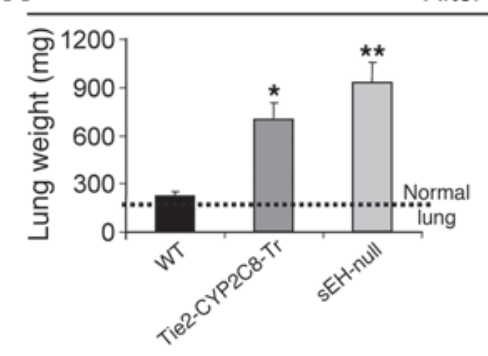

After LLC resection (day 10)
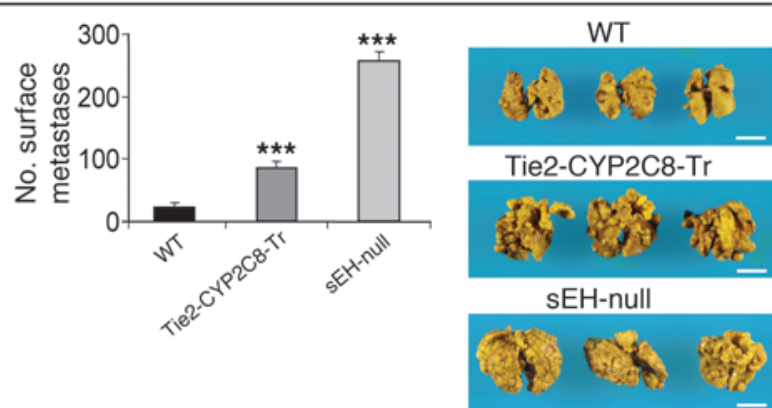

B After LLC resection (day 17)

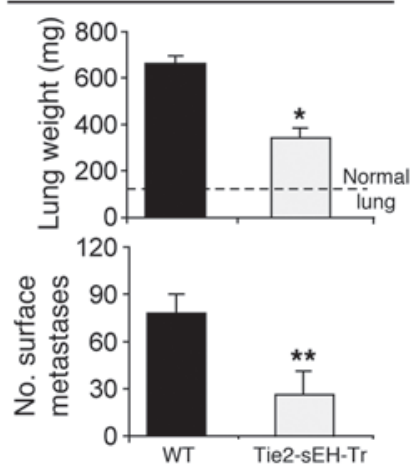

\section{C}
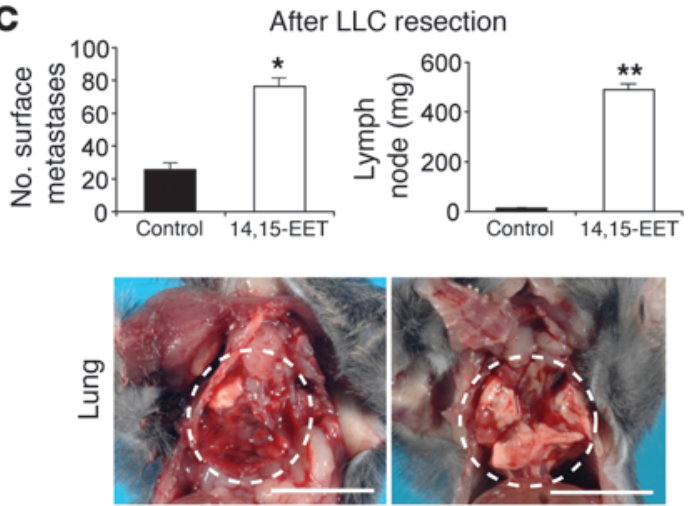

14,15-EET
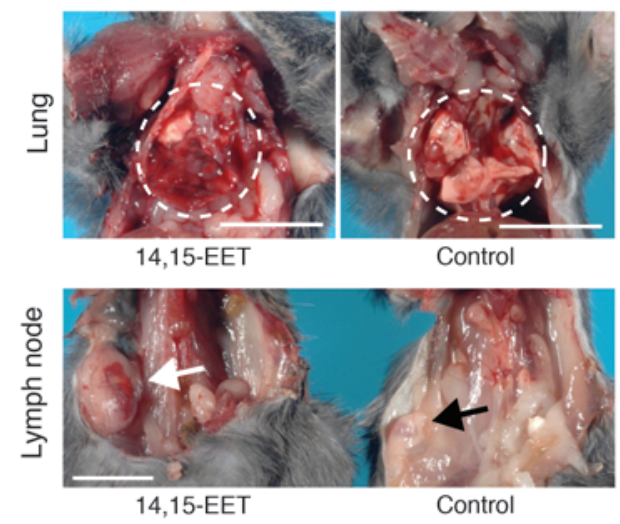

Control

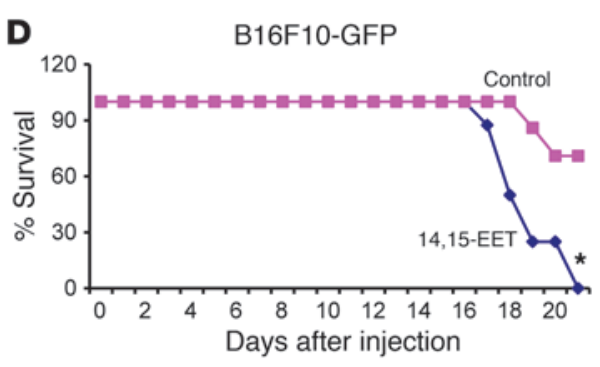

After LLC-GFP resection
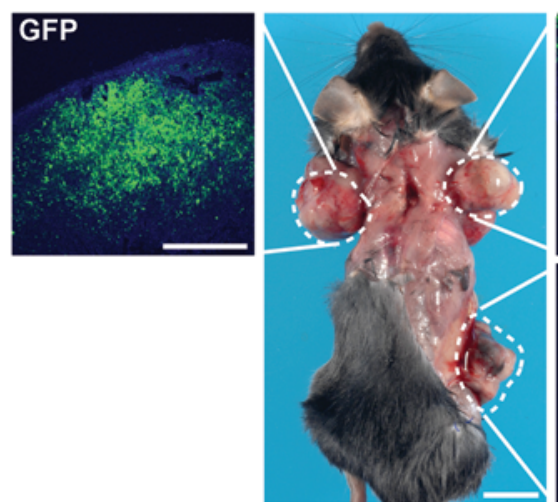

14,15-EET

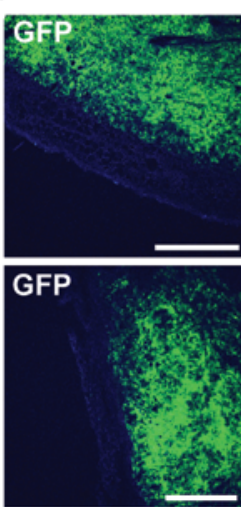

Is:de

\section{B16F10-GFP}
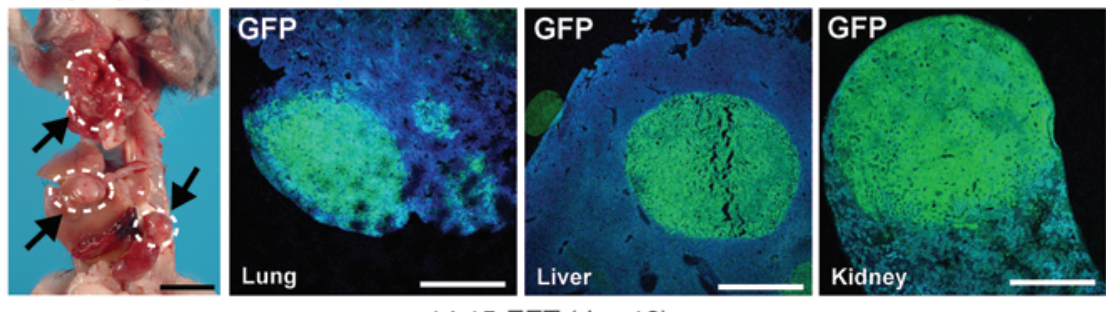

14,15-EET (day 18)

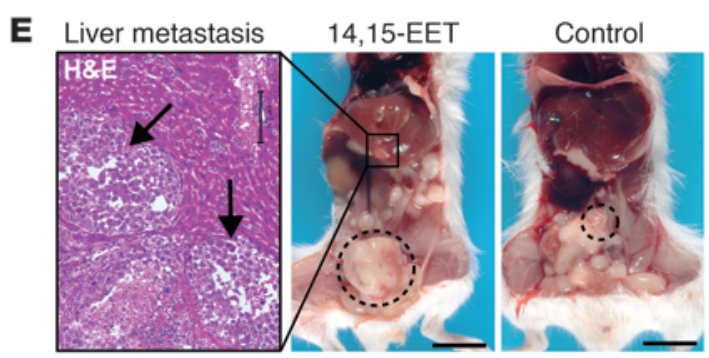




\section{Figure 2}

EETs trigger spontaneous and multiorgan metastasis. (A) Spontaneous LLC metastasis is increased in Tie2-CYP2C8-Tr and sEH-null mice relative to WT 10 days after primary tumor removal (LLC resection). Images show representative lung metastasis in transgenic and WT mice. $n=5$ mice/group; ${ }^{*} P=0.011,{ }^{* *} P=0.004,{ }^{* * *} P \leq 0.001$ versus WT. The experiment was performed 3 times, with similar results. Scale bars: $1 \mathrm{~cm}$. (B) Spontaneous LLC metastasis is decreased in Tie2$\mathrm{sEH}-\mathrm{Tr}$ relative to WT 17 days after primary tumor removal. $n=6$ mice/ group; ${ }^{\star} P=0.001,{ }^{\star \star} P=0.002$. (C) Systemic administration of 14,15 EET $(15 \mu \mathrm{g} / \mathrm{kg} / \mathrm{d})$ via osmotic minipump increases spontaneous LLC lung metastasis and distant axillary and inguinal lymph node metastasis 12 days after LLC resection. Dashed circles indicates metastatic and normal lung; arrows show inguinal lymph node metastasis (white arrow, 14,15-EET; black arrow, control); scale bars: $1 \mathrm{~cm}$. GFP-labeled LLC tumor cells (green) demonstrate bilateral axillary and inguinal lymph node metastasis in mice treated with 14,15-EET 17 days after LLCGFP tumor resection; scale bars: $500 \mu \mathrm{m}$ (for GFP staining). $n=10$ mice/group; ${ }^{*} P=0.001,{ }^{* \star} P \leq 0.001$. (D) 14,15 -EET $(15 \mu \mathrm{g} / \mathrm{kg} / \mathrm{d})$ reduces survival and stimulates multiorgan lung, liver, and kidney metastasis of B16F10-GFP melanoma cells injected intravenously. Dashed circles indicate (top to bottom) representative lung, liver, and kidney metastasis 19 days after injection; scale bar: $1 \mathrm{~cm}$. GFP-labeled melanoma tumor cells (green) confirm lung, liver, and kidney metastasis in mice treated with 14,15-EET; scale bar: $500 \mu \mathrm{m}$ (for GFP staining). $n=7-9$ mice/group; ${ }^{*} P=0.021$. (E) $14,15-E E T(15 \mu \mathrm{g} / \mathrm{kg} / \mathrm{d})$ stimulates liver metastasis in an orthotopic human prostate cancer (PC3M-LN4) model. H\&E-stained section of liver metastasis of mice treated with 14,15-EET; arrows indicate nodules of tumor within hepatic parenchyma; scale bar: $100 \mu \mathrm{m}$. Black circles indicate representative orthotopic prostate tumors; scale bars: $1 \mathrm{~cm} . n=5-6$ mice/group.

To confirm that the atypical lymph node lesions indeed represented metastatic tumors derived from the primary LLC tumor cells, we repeated the metastasis experiments using GFP-labeled LLC tumors in mice administered 14,15-EET systemically. Resection of the primary GFP-LLC tumor again stimulated pronounced axillary and inguinal lymph node masses that contained abundant GFP-positive LLC tumor cells (Figure 2C), thus excluding the possibility that lymph node masses were due to reactive lymphadenopathy. Accordingly, in our other metastasis model, systemic administration of 14,15-EET to mice injected with GFP-labeled B16F10 melanoma cells via tail vein also induced multiorgan metastasis of the injected cells, as indicated by GFP-labeled tumor cells in the lung, liver, and kidney (Figure 2D). Although 14,15-EET induced melanocytic metastases in the lung, the metastases in the liver and kidney were amelanotic (Figure 2D and Supplemental Figure $2 \mathrm{H}$ ), consistent with previous studies indicating that the organ microenvironment influences pigmentation of metastatic murine melanoma (35). Liver metastasis was also achieved by systemic administration of 14,15-EET for 28 days to mice carrying the orthotopic human prostate cancer (PC3M-LN4), whereas control vehicle-treated mice did not exhibit any liver metastasis (Figure 2E). Such metastasis is unexpected in this tumor model, which typically develops paraaortic lymph node metastases, which indeed was consistently observed in the 14,15-EET-treated but only occasionally in the control-treated TRAMP animals (data not shown).

Pharmacological control of EETs in cancer. To confirm the observed effects of exogenous 14,15-EET and to establish the clinical relevance of pharmacological EET modulation, we characterized the effect of EET-modifying drugs in primary tumor and metastasis models. First, we studied compounds that increase EETs by inhibiting sEH. Notably, sEH inhibitors similar to $t \mathrm{AUCB}$, as well as the structurally dissimilar TUPS, are currently being tested in clinical trials for treatment of hypertension $(4,36)$. Treatment of mice with $t$ AUCB, which elevated plasma EET levels up to 3-fold (Supplemental Figure 3A), accelerated primary LLC-GFP tumor growth (Figure 3A). LC/MS/MS analysis of LLC-GFP tumors treated with $t$ AUCB showed no change in EET levels in the tumor tissue (Supplemental Figure 3B). Immunohistochemical analysis of $t$ AUCB-treated LLC-GFP tumors revealed an increase in the number of tumor cells expressing VEGF and a marked increase in microvessels, as stained by the EC marker MECA-32 (Figure 3B and Supplemental Figure 3C). Systemic administration of either tAUCB or TUPS dramatically stimulated lung, liver, and axillary lymph node metastasis in two different murine tumor models (LLC and B16F10 primary tumor resection) (Figure 3C and Supplemental Figure 3D). The sEH inhibitors (TUPS and $t \mathrm{AUCB}$ ) also promoted lymph node metastasis after B16F10 tumor resection to greater than $10 \%-20 \%$ of body weight (Figure 3C). The use of GFPlabeled tumor cells and immunohistochemistry again confirmed that this lymph node enlargement was caused by metastatic tumor cells (Supplemental Figure 3, E and F).

Conversely, mice with established LLC tumors treated with the putative EET receptor antagonist 14,15-EEZE demonstrated reduced primary LLC growth, prolonged survival in the LLC resection metastasis model, and reduced plasma VEGF levels (Figure 3D). While the receptor(s) for EETs have not yet been identified (4), EET antagonists are the most specific reagents available for pharmacologic suppression of EET activity $(37,38)$ and have been used to antagonize the biological effects of EETs in other disease models (39-43). To determine whether an EET antagonist could prevent EET-induced metastasis, we co-administered the EET antagonist 14,15-EEZE-mSI with 14,15-EET following LLC primary tumor resection. The EET antagonist drastically reduced lung metastasis and prevented the macroscopic liver and lymph node metastasis typically induced by 14,15-EET (Figure 3E) without apparent toxic effects. In addition, systemic administration of the EET antagonist 14,15-EEZE inhibited lung and lymph node metastases in Tie2-CYP2C8-Tr and sEH-null mice (Supplemental Figure 3G). Vessel density decreased in B16F10-GFP tumors treated with 14,15-EEZE (Supplemental Figure 3H). Thus, pharmacological modulation of EET levels recapitulates the results obtained in genetically altered mice with respect to both stimulation and suppression of primary tumor and metastatic growth.

EETs require VEGF for tumor progression. Since the receptor(s) for EETs are unknown (4), it is difficult to elucidate the cellular and molecular targets of EETs in promoting cancer. We examined possible cellular processes that might mediate the effects of EETs on tumor growth and metastasis. Increased metastasis in part results from increased EC migration, a critical component of the metastatic cascade $(44,45)$. ECs isolated from the aortas of Tie2-sEH-Tr mice, which have endothelium-specific staining of sEH (Supplemental Figure 4A), exhibited decreased migration on collagen substrates when compared with aortic ECs from WT mice (Figure 4A). In contrast, ECs from Tie2-CYP2J2-Tr and Tie2-CYP2C8-Tr mice exhibited increased migration relative to WT ECs (Figure 4A). tAUCB and TUPS had no significant effects on basal endothelial migration but increased VEGF-mediated endothelial migration 2- to 3-fold (Figure $4 \mathrm{~A})$. In contrast, the putative EET receptor antagonist 14,15-EEZE inhibited EC migration in a dose-dependent fashion but had no significant effect on migration of LLC tumor cells (Figure 4B). 
A

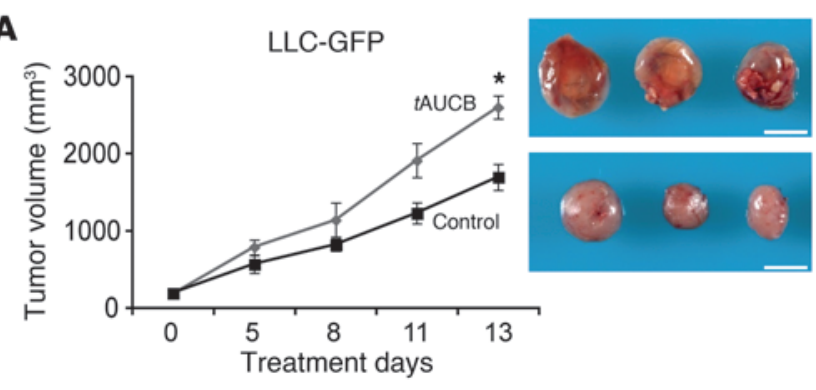

B
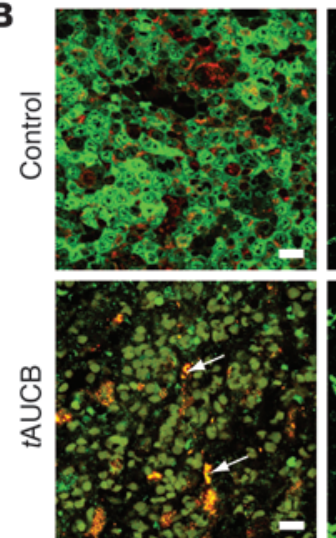

GFP (tumor) VEGF
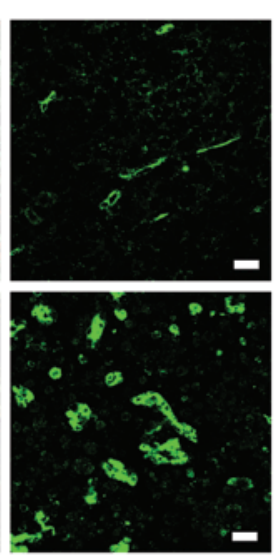

MECA-32

C After B16F10 resection
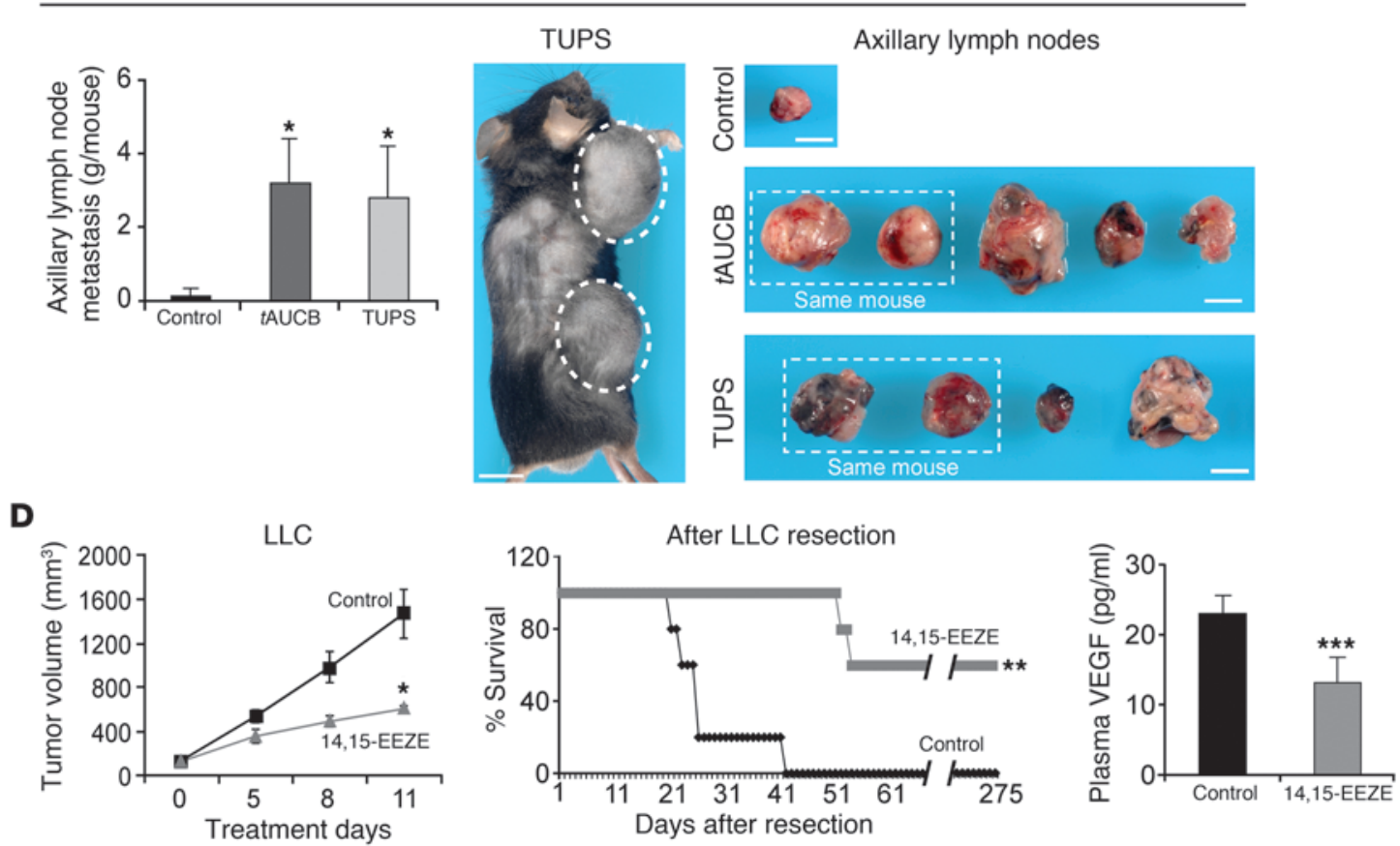

E
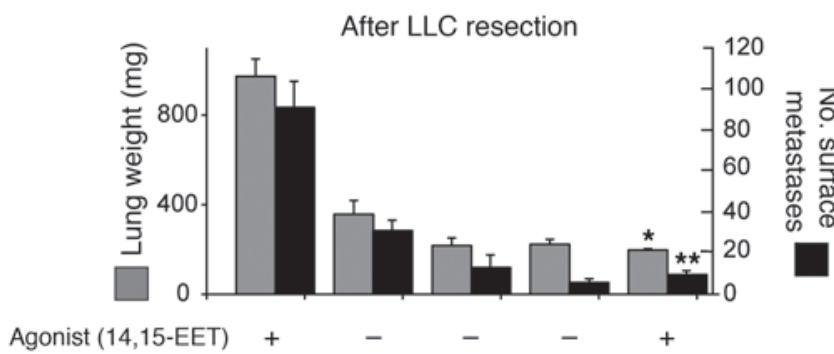

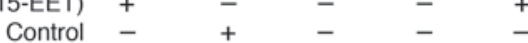

$\begin{array}{llllll}\text { Metabolite }(14,15-\mathrm{DHET}) & - & - & + & - & -\end{array}$

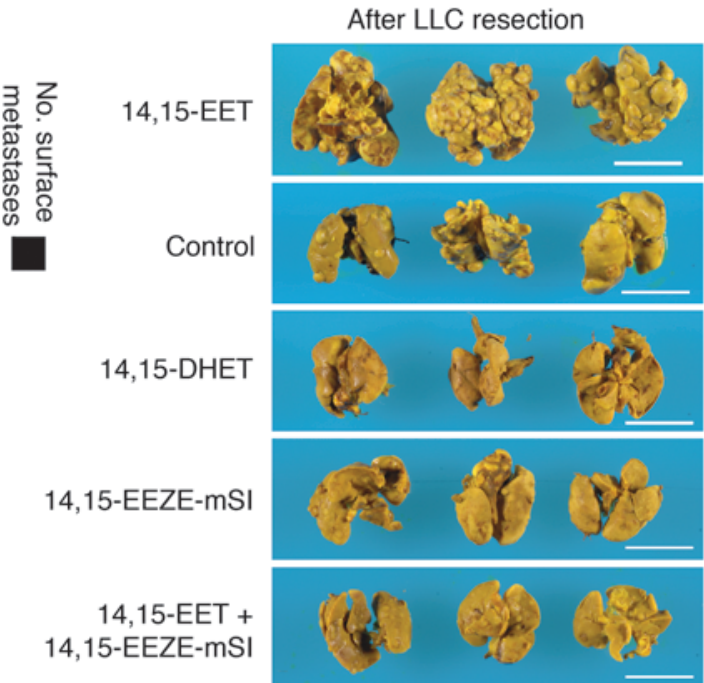




\begin{abstract}
Figure 3
Pharmacological modulation of EET levels controls primary tumor growth and metastasis. (A) Systemic administration of an sEH inhibitor (tAUCB) stimulates primary LLC-GFP tumor growth. Images show representative tumors after 13 days of treatment. $n=6$ mice/group; ${ }^{\star} P=0.007$. Scale bar: $1 \mathrm{~cm}$. (B) Immunofluorescence double staining for VEGF and GFP (tumor cells) shows increased tumor cell expression of VEGF in LLC-GFP tumor cells from tAUCB- versus vehicletreated mice. Green, GFP-stained tumor cells; red, VEGF-containing cells. Colocalization of red and green fluorescence (yellow) indicates tumor cells expressing VEGF (arrows). tAUCB-treated tumors have an increase in MECA-32-positive ECs (green). Scale bars: $20 \mu \mathrm{m}$. (C) Systemic administration of $t A U C B$ and TUPS (10 mg/kg/d each) increases spontaneous B16F10 axillary lymph node metastasis 21 days after B16F10 resection. Representative axillary lymph nodes after 21 days of treatment are shown. Scale bars: $1 \mathrm{~cm} . n=6 \mathrm{mice} /$ group; * $P=0.029$ versus control. (D) The EET antagonist $14,15-$ EEZE (0.21 $\mathrm{mg} / \mathrm{mouse}$ ) inhibits primary LLC growth (left panel), prolongs survival (middle panel), and reduces plasma VEGF levels (right panel) in a spontaneous LLC lung metastasis model. $n=5$ mice/group; ${ }^{\star} P=0.017,{ }^{* \star} P=0.035,{ }^{* \star \star} P=0.044$. (E) The EET antagonist 14,15 EEZE-mSI (0.21 mg/mouse) inhibits 14,15-EET-induced $(15 \mu \mathrm{g} / \mathrm{kg} / \mathrm{d})$ spontaneous LLC metastasis. The stable EET metabolite 14,15-DHET $(15 \mu \mathrm{g} / \mathrm{kg} / \mathrm{d})$ does not stimulate metastasis. Representative photographs on day 12 after LLC resection are shown. Scale bars: $1 \mathrm{~cm}$. $n=5$ mice/group; ${ }^{*} P<0.001,{ }^{* \star} P=0.003$ versus $14,15-\mathrm{EET}$.
\end{abstract}

Both Tie2-CYP2C8-Tr and sEH-null mice exhibited a significant increase in plasma levels of VEGF when compared with WT mice (Figure 4C). 14,15-EET upregulated the VEGF receptor VEGFR2 but not VEGFR1 in LLC tumors in comparison to size-matched control tumors (Figure 4C), suggesting the typical VEGF-VEGFR2 interaction reported for ECs (46). Immunohistochemistry of 14,15-EET-treated LLC tumors showed mild to moderate VEGFR2 expression in ECs, leukocytes (including macrophages), and a few tumor cells (Supplemental Figure 4, B and C). To determine whether VEGF-stimulated angiogenesis plays a functional role in EET-mediated tumor growth, we depleted VEGF by expressing a soluble form of VEGF receptor 1 (sFlt) using an adenoviral delivery system (47). In WT mice, which have low systemic VEGF levels, tumor growth was approximately half that in the high-EET mice (Figure 4D), and VEGF depletion had no significant effect on primary B16F10 melanoma growth (Figure 4D). In contrast, in Tie2-CYP2J2-Tr and sEH-null mice, where EET and VEGF levels were higher than in WT mice, VEGF depletion suppressed the accelerated B16F10 melanoma tumor growth by up to $80 \%$ at day 19 after tumor injection (Figure 4D). In fact, $t A U C B$ was unable to promote tumor growth and metastasis in mice depleted of VEGF by Ad-sFlt (Figure 4E and Supplemental Figure 4D). Thus, EETs require VEGF for tumor progression.

Interestingly, B16F10 tumors in mice expressing sFlt eventually escaped the VEGF depletion, suggesting that changes in other regulators of tumor angiogenesis, such as thrombospondin-1 (TSP1), which is an angiogenesis inhibitor (48), may also play a role. Indeed, plasma from tumor-bearing Tie2-CYP2C8-Tr, Tie2-CYP2J2-Tr, and sEH-null mice exhibited a pronounced reduction in the levels of TSP1 at day 13 after injection when compared with WT mice (Figure 4F), suggesting that this effect may contribute to the tumorpromoting activity of EETs and may help to overcome the neutralization of VEGF. Stromal TSP1 expression was markedly decreased in three different tumors (LLC, B16F10 melanoma, and T241 fibro- sarcoma) in Tie2-CYP2C8-Tr and sEH-null mice when compared with WT mice (Supplemental Figure 4E). In addition, 14,15-EET specifically decreased TSP1 production in fibroblasts but had no effect on TSP1 production in tumor and ECs in vitro (Supplemental Figure 4F). To evaluate the potential role of TSP1 suppression as a mediator of EET-induced tumor angiogenesis, we treated TSP1deficient (TSP1-null) mice with the EET antagonist 14,15-EEZE and found that tumor suppression by the EET antagonist was significantly diminished, by $62 \%$, in WT mice (Figure 3D) to $24 \%$ in TSP1-null mice (Figure 4G). Thus, regulation of TSP1 by EET antagonists may contribute to their tumor-suppressive activity.

Given the elevated plasma VEGF levels in Tie2-CYP2J2-Tr and sEH-null mice, we next asked whether EETs had an effect on VEGF production. We found that 14,15-EET increased VEGF production by tumor cells and ECs in vitro but had no effect on VEGF production in fibroblasts (Supplemental Figure 4G). VEGF can be produced by tumor stromal cells (49). To identify the source of VEGF, we analyzed LLC tumors in VEGF-LacZ-Tr mice, in which the LacZ reporter was introduced into the VEGF locus (50). Interestingly, the endothelium and other stromal cells of LLC tumors grown in VEGF-LacZ-Tr mice systemically treated with 14,15-EET stained positively for the LacZ product $\beta$-galactosidase, whereas this marker for VEGF production was absent in the stroma of sizematched control tumors of nontreated mice (Figure 5A).

Source of EETs in advanced tumors. If EETs stimulate tumor angiogenesis, what causes EET production to rise during progressive tumor growth? In humans, expression of sEH (which metabolizes EETs) is downregulated in hepatic and renal cancer (51). To determine whether the loss of the enzyme sEH indeed occurs in the endothelium and if respective changes in CYP epoxygenase and sEH expression contribute to tumor progression via increase in EETs, we analyzed isolated tumor ECs and normal "quiescent" ECs (52) and measured expression of sEH, CYP2C, and CYP2J proteins. While CYP2C and CYP2J levels were similar in the two populations of ECs, we observed a significant decrease in sEH in tumor ECs in comparison to normal ECs (Figure 5B and Supplemental Figure $4 G$ ). In contrast, all murine tumor cell lines that we examined expressed sEH, CYP2C, and CYP2J in vitro, except for LLC, which appeared to be CYP2J-negative (Supplemental Figure 4H). By contrast, examination of LLC tumor lysates revealed that expression of sEH, but not CYPs, decreased with tumor progression (Figure 5B). Similarly, sEH expression was suppressed in liver metastasis of B16F10 melanoma tumors compared with adjacent normal liver tissue (Figure 5B). Collectively, these findings indicate that tumor growth and progression are associated with decreased sEH expression, most notably in the tumor endothelium, suggesting that downregulation of sEH may account for the increased EETs and subsequent stimulation of angiogenesis in progressing tumors.

Endothelial EETs act at secondary sites in metastasis. High endothelial EET levels induced a dramatic increase in number, size, and spread of distant metastases. To determine whether the systemic modulation of EET levels facilitates cancer cell dissemination at the primary tumor site (by promoting extravasation and migration) or at the metastatic site (by promoting homing, colonization, dormancy escape, and survival), we utilized a parabiosis model with a shared circulatory system $(53,54)$, with a tumor-bearing (donor) mouse with high EET levels (Tie2-CYP2C8-Tr or sEH-null) conjoined to a non-tumor-bearing (recipient) mouse with normal or low endothelial EET levels (WT or Tie2-sEH-Tr, respectively). Other configurations served as controls. The sharing of humoral factors 
A
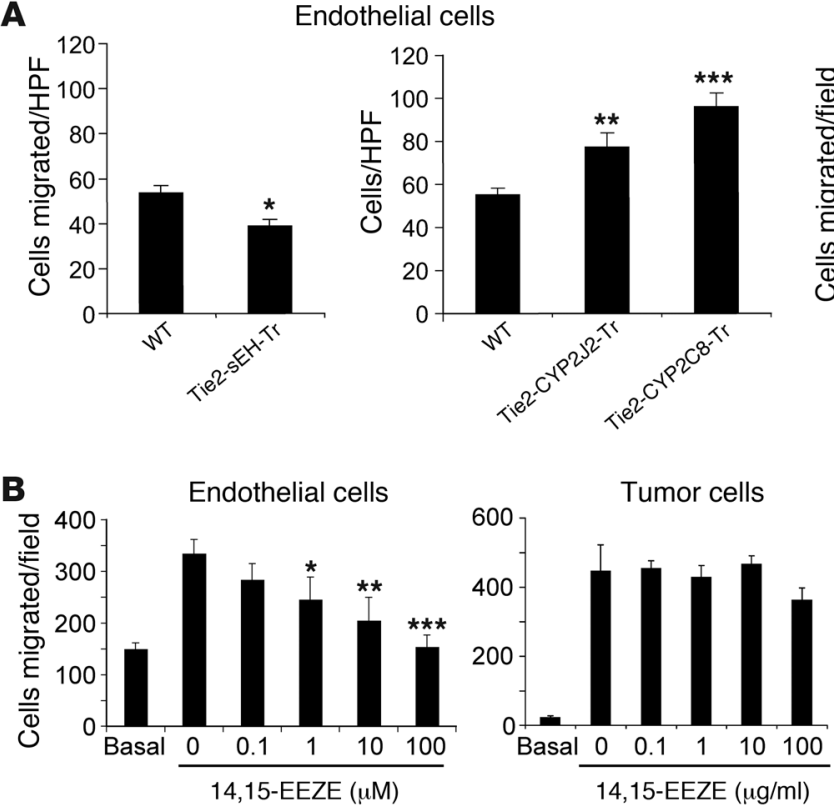

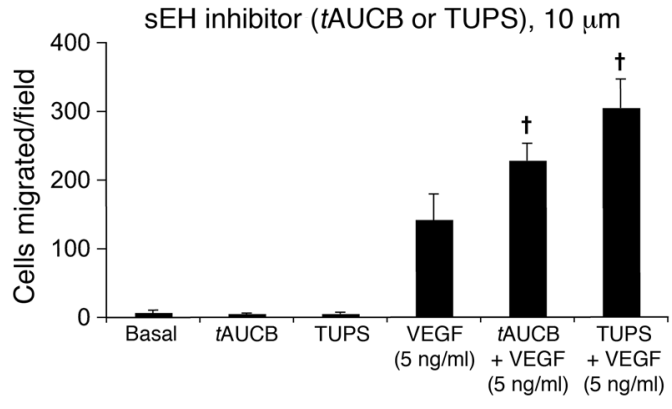

C
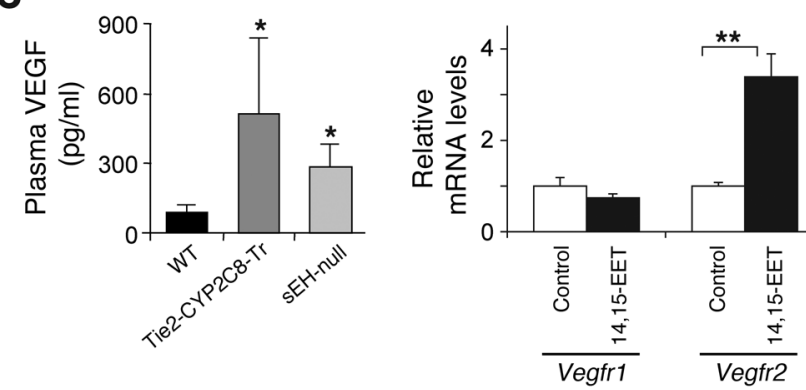

sEH-null

$\mathbf{E}$

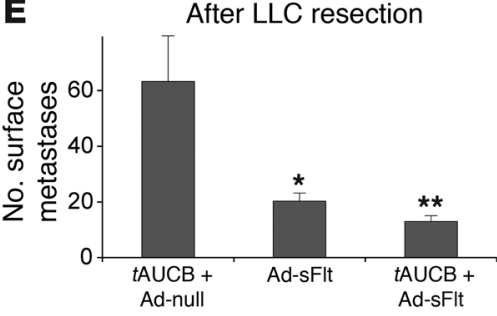

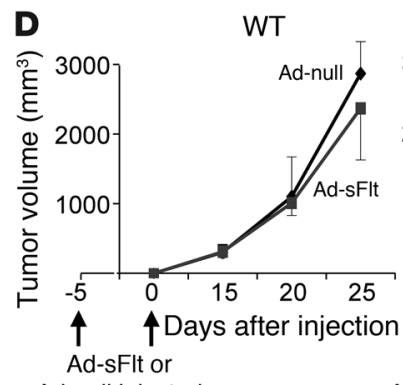

Ad-null injected

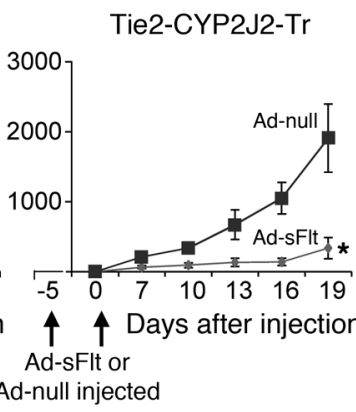

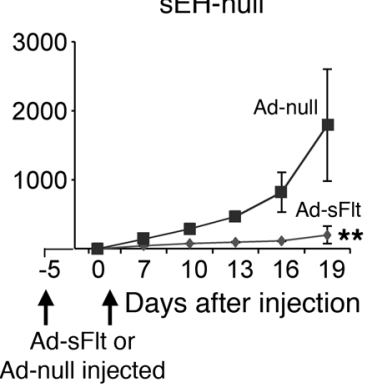

Ad-sFlt or

Ad-null injected
$\mathbf{F}$

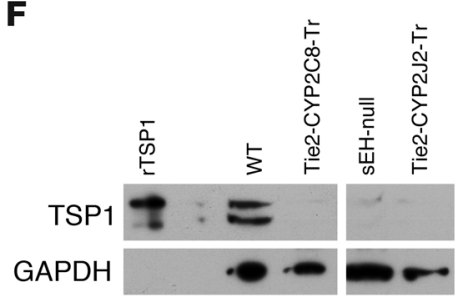

G

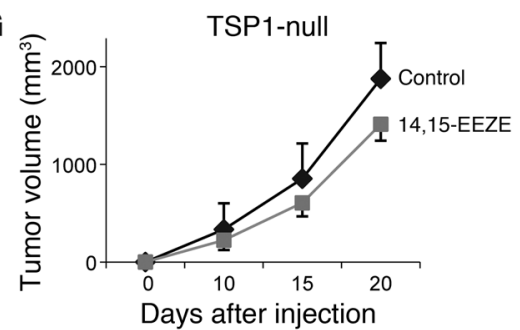

Figure 4

Pro-tumorigenic activity of endothelium-derived EETs is mediated by VEGF induction and loss of TSP1. (A) EC migration is decreased in Tie2-sEH-Tr mice (left panel) but increased in Tie2-CYP2J2-Tr and Tie2-CYP2C8-Tr relative to WT mice (middle). tAUCB and TUPS stimulate VEGF-mediated endothelial migration (right). $n=3-4 /$ group; ${ }^{*} P=0.0139,{ }^{* *} P=0.029$, and ${ }^{* * *} P<0.001$ versus WT; ${ }^{*} P<0.05$ versus VEGF alone. (B) 14,15-EEZE inhibits VEGF-induced EC (left panel) but not tumor cell (LLC) migration (right). $n=3-4 /$ group; ${ }^{*} P=0.038,{ }^{* *} P=0.012$, ${ }^{* * \star} P<0.001$ versus control. (C) VEGF ELISA of plasma of Tie2-CYP2C8-Tr and sEH-null mice 17 days after B16F10 resection shows an increase in VEGF levels. $n=5 /$ group (left panel). Systemic administration of 14,15-EET regulates Vegfr2 mRNA levels in primary LLC tumors in mice compared with size matched control tumors, but has no effect on Vegfr 1 mRNA levels (right). $n=5 /$ group. ${ }^{*} P<0.05$ versus WT; ${ }^{* *} P=0.0095$ versus control. (D) VEGF depletion with Ad-sFlt suppresses B16F10 tumor growth in Tie2-CYP2J2-Tr and sEH-null but not in WT mice. Plasma levels of sFlt1 on day 10 were 35,999 $\pm 10,225 \mathrm{pg} / \mathrm{ml}$ (Tie2-CYP2J2-Tr) and 36,680 $\pm 1,308 \mathrm{pg} / \mathrm{ml}$ (sEH-null). $n=5$ mice/group; ${ }^{*} P=0.016,{ }^{* *} P=0.023$. (E) tAUCB does not promote spontaneous LLC metastasis in mice depleted of VEGF with Ad-sFlt (tAUCB + Ad-sFIt). $n=5$ mice/group; ${ }^{*} P=0.004,{ }^{*} P=0.002$ versus $t A U C B+A d-$ null. $(\mathbf{F})$ The angiogenesis inhibitor TSP1 is downregulated in plasma of Tie2CYP2C8-Tr, sEH-null, and Tie2-CYP2J2-Tr relative to WT mice on day 13 after LLC injection. rTSP1, recombinant TSP1. (G) 14,15-EEZE $(0.21 \mathrm{mg} / \mathrm{mouse})$ does not significantly inhibit primary LLC tumor growth in TSP1 null mice. $n=5$ mice/group. 
A Control
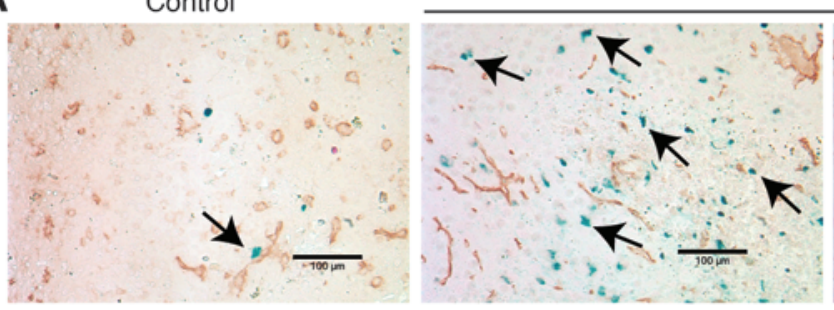

B

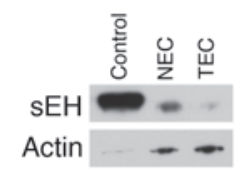

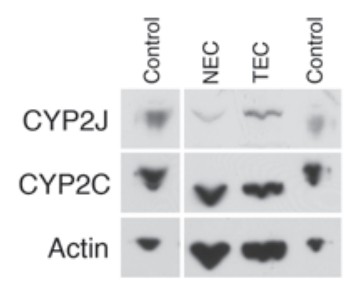

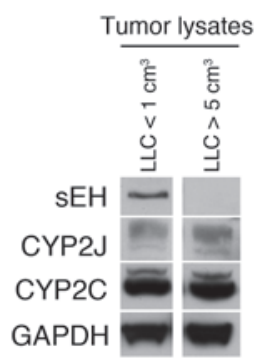

14,15-EET

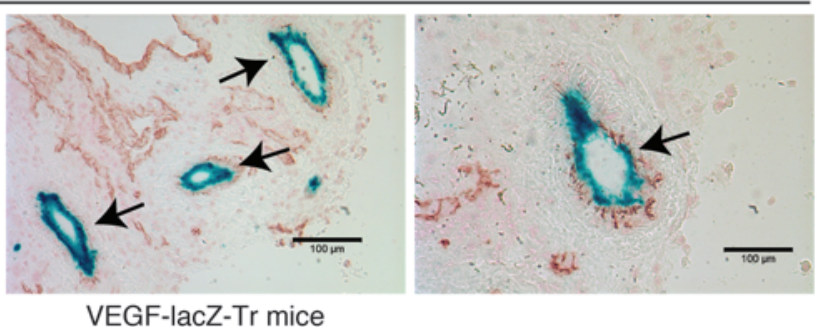

VEGF-lacZ-Tr mice

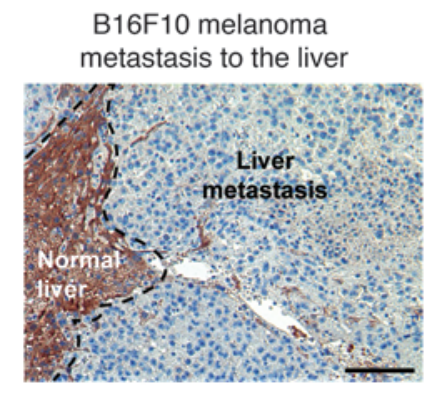

SEH

\section{Figure 5}

Pro-tumorigenic activity of EETs is mediated by VEGF production in the tumor stroma and loss of sEH. (A) LLC tumors in VEGF-LacZ-Tr mice treated with 14,15-EET $(15 \mu \mathrm{g} / \mathrm{kg} / \mathrm{d})$ show $\beta$-galactosidase staining (marker of VEGF production) in tumor endothelium and stromal fibroblasts (arrows). Scale bars: $20 \mu \mathrm{m}$. (B) Expression of sEH, but not CYP2J and CYP2C, is downregulated in tumor (TEC) versus normal (NEC) ECs (two left panels). Control tissue: mouse liver. Expression of SEH, but not CYP2J and CYP2C, is downregulated in tumor lysates from larger LLC tumors $\left(>5 \mathrm{~cm}^{3}\right)$ versus smaller LLC tumors $\left(<1 \mathrm{~cm}^{3}\right)$ (third panel). sEH expression (brown staining) is also downregulated in B16F10 melanoma liver metastasis compared with normal adjacent liver (far right panel). Scale bar: $100 \mu \mathrm{m}$.

in the parabiotic circulation was confirmed using Evans blue dye as a tracer (Supplemental Figure 5, A and B). Results of the experiments are summarized in Tables 1 and 2 and Supplemental Figure 5C. In the control configuration - in which two "high-EET" mice were parabiosed but only one carried the primary tumor - liver, lung, and lymph node metastases occurred in both parabionts, demonstrating that parabiosis itself did not interfere with EETpromoted metastasis (Tables 1 and 2, cases b, c, and i). Moreover, the genotype of the tumor-bearing donor mouse determined the growth of the primary tumor (in the donor mouse), regardless of the genotype of the recipient mouse. Specifically, sEH-null (highEET) recipients could not rescue the tumor phenotype of Tie2sEH-Tr (low-EET) donors (Table 1, case f). It is possible that the high EET levels in the plasma of sEH-null mice, which are shared by both partners, were not sufficient to rescue tumor growth in the Tie2-sEH-Tr mice, where the sEH enzyme acts as a local sink to keep EET levels in the endothelium low. In the key configuration, a low-EET recipient attached to a high-EET donor failed to produce metastasis (Table 2, cases $\mathrm{j}$ and $\mathrm{k}$ ), demonstrating that a high-EET-producing endothelium is critical at the metastatic site for lung, liver, and lymph node metastases to occur. Importantly, adoptive transfer of whole blood from low-EET recipient parabionts (Tie2-sEH-Tr), which exhibited no metastasis, caused metastatic disease when inoculated into nonparabiotic high-EET (Tie2-CYP2C8-Tr) mice, confirming that the low-EET parabiont had circulating tumor cells. Indeed, 28 days after injection, 100\% of the WT recipients of such blood transfer survived, while $50 \%$ of the Tie2-CYP2C8-Tr blood transfer recipients died of metastasis (Supplemental Figure 5D). Immunofluorescence staining of histo- logical sections of tumors removed from the high-EET parabionts showed increased tumor EC proliferation and increased VEGF production (Supplemental Figure 5E). Together, these findings document the requirement for high EET levels in the endothelium of the metastatic site in order for metastasis to occur.

\section{Discussion}

We demonstrate that exogenous administration of EETs in vivo promote tumor growth, as well as dramatically enhancing metastatic spread in several tumor models known to metastasize poorly. Specifically, in Tie2-CYP transgenic mice, which are engineered to raise endothelial EET levels, tumors that rarely metastasize exhibit extensive metastatic spread into a range of organs, including lung, axillary lymph nodes, liver, and kidney. Exogenous administration of EETs induce multiorgan metastasis and tumor dormancy escape in a variety of transplantable and genetically engineered cancer models to a high degree, providing what we believe to be the first in vivo demonstration that pharmacological modulation of these lipid autacoids affects tumor growth.

EETs promote metastasis by triggering secretion of VEGF by the endothelium, which we show to be critical for EETs' cancerstimulating activity. We also demonstrate that stimulation of metastasis by EETs is due to their action at the secondary (metastasis) site and not due to their effect on the cells of the primary tumor. We arrived at this insight by dissecting the two steps of metastasis using a series of parabiosis experiments in which mice transgenically modified to produce either low or high levels of EET in the endothelium were surgically cojoined. Using parabiosis, we have delineated and identified the distinct roles of stromal 
Table 1

Parabiosis donor/recipient constellations on primary tumor growth (LLC)

\begin{tabular}{cccc} 
Case & $\begin{array}{c}\text { Parabiotic donor } \\
\text { (site of primary tumor) }\end{array}$ & Recipient (site) & $\begin{array}{c}\text { Primary tumor volume } \\
\left(\mathbf{m m}^{\mathbf{3}} \mathbf{)}\right.\end{array}$ \\
a & WT & WT & $1,565 \pm 170$ \\
b & SEH-null $\rightarrow$ EET high & sEH-null $\rightarrow$ EET high & $6,869 \pm 311^{\mathrm{A}}$ \\
c & Tie2-CYP2C8-Tr $\rightarrow$ EET high & Tie2-CYP2C8-Tr $\rightarrow$ EET high & $5,632 \pm 1,004 \mathrm{~A}$ \\
d & SEH-null $\rightarrow$ EET high & Tie2-sEH-Tr $\rightarrow$ EET low & $6,123 \pm 505^{\mathrm{A}}$ \\
e & Tie2-CYP2C8-Tr $\rightarrow$ EET high & WT $\rightarrow$ EET low & $5,046 \pm 526^{\mathrm{A}}$ \\
f & Tie2-sEH-Tr $\rightarrow$ EET low & sEH-null $\rightarrow$ EET high & $1,333 \pm 183$ \\
g & Tie2-sEH-Tr $\rightarrow$ EET very low & Tie2-sEH-Tr $\rightarrow$ EET very low & $1,031 \pm 186$ \\
\hline
\end{tabular}

Parabiosis shows that EET-stimulated tumor growth depends on genotype of tumor-bearing parabiont (donor). Parabiosis was performed for 4-5 pairs per configuration and mean tumor volume \pm SEM determined. ${ }^{A} P<0.05$ versus cases $a, f$, and $g$.

EET levels in promoting or inhibiting growth of the primary tumor and at the point of metastasis.

Although we show that the endothelium, acting as a source of local EETs, is a critical regulator of primary tumor growth and metastasis, our studies with exogenously administrated 14,15-EET indicate that high levels of systemic EETs can also stimulate tumor growth and metastasis. The levels of EETs after adenoviral CYP expression increase approximately 3 - to 5 -fold in vitro (11). Treatment with 14,15-EET via osmotic minipump in our studies yielded a plasma steady-state EET concentration of approximately $3.0 \mathrm{ng} / \mathrm{ml}$, twice that observed in sEH null mice $(\sim 1.5 \mathrm{ng} / \mathrm{ml})$, which have high levels of systemic EETs, and 15- to 30-fold higher than that in Tie2CYP2J2-Tr and Tie2-CYP2C8-Tr mice (100-200 pg/ml). This may explain why systemic exogenous 14,15 -EET was able to stimulate tumor growth, whereas in the parabiosis studies, the high-EET partner (e.g., sEH-null mouse) failed to rescue tumor growth in the lowEET partner. Plasma EET levels were likely diluted in the parabiosed low-EET partner to levels that approximate those in WT mice.

Our work demonstrates a number of important findings on lipid autacoids and in particular EETs in tumor biology. We establish a connection between the disparate fields of lipid autacoids and tumor biology. By manipulating EET levels in vivo, as opposed to CYP levels or activity (11), we demonstrate the dramatic metastatic potential of EETs on low-metastasizing tumors, thereby opening an avenue for metastasis research, which has suffered from the scarcity of spontaneous metastasis models. EETs in the endothelium may also be a key paracrine mediator of the tumor-promoting role of the stroma, revealing a trophic function for the endothelium in promoting tumor growth in addition to its role in providing circulation. Inflammatory cells such as macrophages may also contribute to EET-mediated tumor growth. We also demonstrate that $\mathrm{SEH}$ inhibitors, which elevate endogenous EET levels, promote primary tumor growth and metastasis. Our findings are of potential clinical relevance because drugs that raise EET levels are now in clinical trials for the treatment of hypertension and are being considered for long-term use in other medical conditions $(4,5)$. Our finding that $\mathrm{sEH}$ inhibitors pro- mote tumor growth, notably explosive metastatic growth from presumably dormant disseminated tumor cells, raises significant concerns about the use of sEH inhibitors, especially their chronic use, which may adversely affect cancer patients. Dormant tumors have been identified at autopsy in normal adults who died of trauma and without prior history of clinical evidence of cancer, including $39 \%$ for in situ breast carcinoma, $46 \%$ for in situ prostate cancer, and 36\% for thyroid carcinoma in people aged $50-70(55,56)$. The factors that regulate tumor dormancy and escape from it are poorly understood (57). The escape is critically dependent on the induction of angiogenesis $(22,25,26,44)$. Our data suggest that the increase in EET levels, due to downregulation of sEH, may support the angiogenic switch in part by an increase in VEGFR2 and loss of thrombospondin.

We also demonstrate the ability of EET antagonists to inhibit tumor growth and metastasis. These results could pave the way for a new strategy for the prevention and treatment of metastatic disease - i.e., inhibition of EET bioactivity. Specific EET antagonists, inhibitors of endothelial CYP epoxygenases, or the overexpression of EET-metabolizing enzymes may represent new strategies for the treatment of angiogenic diseases, including cancer. Manipulation of the EET system is thus a double-edged sword, and further studies are needed to carefully evaluate the benefits as well as the risks in the clinical modulation of these lipid mediators.

\section{Methods}

Generation of Tie2-CYP2J2, Tie2-CYP2C8, and Tie2-sEH transgenic mice. Transgenic mice expressing human CYP2J2, CYP2C8, or sEH in ECs were developed on a C57BL/6 background. The human CYP2J2 (GenBank NM_000775), CYP2C8 (NM_000770), and sEH (NM001979) cDNA sequences were subcloned downstream of the murine Tie2 promoter and upstream of the Tie2 full enhancer sequences (provided by Thomas N. Sato, University of Texas Southwestern Medical Center) to drive endothelial expression, as described previously $(15,18,58)$. Linearized constructs were excised from the vector backbone, agarose gel purified, microinjected into pronuclei of single-cell C57BL/6J mouse embryos, and implanted into pseudopregnant mice. Hemizygous founder pups were identified via PCR genotyping of genomic DNA. Breeding involved crossing hemizygous Tie2-

\section{Table 2}

Parabiosis donor/recipient constellations on spontaneous LLC metastasis

$\begin{array}{lccc}\text { Case } & \begin{array}{c}\text { Parabiotic donor } \\ \text { (site of primary tumor) }\end{array} & \text { Recipient (site) } & \begin{array}{c}\text { No. surface lung } \\ \text { metastasis in recipient }\end{array} \\ \text { h } & \text { WT } & \text { WT } & \text { No mets } \\ \text { i } & \text { Tie2-CYP2C8-Tr } \rightarrow \text { EET high } & \text { Tie2-CYP2C8-Tr } \rightarrow \text { EET high } & 27 \pm 4 ; 3 \text { of 4, liver mets; } \\ & & & 3 \text { of 4, LN mets } \\ \text { j } & \text { Tie2-CYP2C8-Tr } \rightarrow \text { EET high } & \text { WT } \rightarrow \text { EET low } & \text { No mets } \\ \text { k } & \text { Tie2-CYP2C8-Tr } \rightarrow \text { EET high } & \text { Tie2-sEH } \rightarrow \text { EET very low } & \text { No mets }\end{array}$

Parabiosis shows that metastasis requires EET-producing endothelium at the metastatic site in the recipient parabiont. Parabiosis was performed for 4-5 pairs per configuration and mean number of metastases (mets) \pm SEM determined. ${ }^{A} P<0.05$ versus cases $\mathrm{h}$, j, and $\mathrm{k}$. 
CYP2J2-Tr, Tie2-CYP2C8-Tr, and Tie2-sEH-Tr founder mice with C57BL/6 WT mice in order to obtain heterozygous $\operatorname{Tr}(+/-)$ and WT littermates (-/-) at an approximate ratio of 1:1. All studies used adult $\mathrm{F}_{2}-\mathrm{F}_{7}$ Tie2-CYP2J2- $\mathrm{Tr}$, Tie2-CYP2C8-Tr, Tie2-sEH-Tr, and WT littermates as controls, were completed in accordance with the NIH Guide for the Care and Use of Laboratory Animals, and were approved by the Animal Care and Use Committee of NIEHS and Children's Hospital, Boston.

Materials. LLC, LLC-GFP, B16F10 melanoma, and T241 fibrosarcoma cells were cultured as described previously (32). 14,15-EET and 14,15-DHET were obtained from Cayman Chemical. 14,15-EET, 14,15-DHET, or vehicle was administered intraperitoneally via osmotic minipump (Alzet) at a dose of $15 \mu \mathrm{g} / \mathrm{kg} / \mathrm{d}$. The sEH inhibitors ( $t$ AUCB and TUPS) were administered orally by gavage in an aqueous solution of $10 \%$ DMSO in $0.5 \%$ methylcellulose, whereas control mice received vehicle. After the ethanol was evaporated under a stream of nitrogen gas, EET antagonists 14,15-EEZE and 14,15EEZE-mSI were administered orally by gavage in an aqueous solution in $0.5 \%$ methylcellulose, while control mice received vehicle.

Tumor xenograft and metastasis studies. All animal studies were reviewed and approved by the Animal Care and Use Committee of Children's Hospital, Boston. Six-month-old male transgenic and WT mice on a pure C57BL/6 background were used. LLC, B16F10 melanoma, and T241 fibrosarcoma tumor cells were injected as described previously (28). For metastasis studies, LLC or B16F10 melanoma tumors were resected 14-21 days after implantation as described (31). B16F10 melanoma cells were injected intravenously as described (28). For pharmacological modulation of EETs, tumors in male, 6-week-old C57BL/6J mice (The Jackson Laboratory) were allowed to reach $100 \mathrm{~mm}^{3}$ before $\mathrm{sEH}$ inhibitors (tAUCB and TUPS), EET antagonists (14,15-EEZE and 14,15-EEZE-mSI), or vehicle were administered by daily gavage for 11-21 days. For metastasis studies, LLC or B16F10 melanoma tumors were resected 17 days after injection. After LLC or B16F10 melanoma resection, mice were treated with sEH inhibitors (tAUCB and TUPS), EET antagonists (14,15-EEZE and 14,15-EEZE-mSI), or vehicle. In VEGF-LacZ-Tr mice, 14,15-EET (Cayman Chemical, $30 \%$ in $\mathrm{EtOH}$ ) or vehicle was administered intraperitoneally via osmotic minipump (Alzet) at a dose of $15 \mu \mathrm{g} / \mathrm{kg} / \mathrm{d}$ on the day of LLC tumor injection. In TRAMP mice (129S background, The Jackson Laboratory), 14,15-EET or vehicle was administered intraperitoneally via osmotic minipump (Alzet) at a dose of $15 \mu \mathrm{g} / \mathrm{kg} / \mathrm{d}$ when mice were $4-6$ weeks of age, and osmotic minipumps were changed once a month for 4 months. For LLC, tumor cells were injected at $1 \times 10^{6}, 1 \times 10^{5}, 1 \times 10^{4}$, and $1 \times 10^{3}$ cells, and 14,15EET or vehicle osmotic pumps were implanted on the day of injection and changed every 2 weeks for 28 days. For orthotopic human prostate cancer, $2 \times 10^{5}$ PC $3 \mathrm{M}$-LC4 cells were injected in 6-week-old male SCID mice $(\mathrm{MGH})$ as described previously (20). For human liposarcoma, clone 4 (nonangiogenic) cells were injected into 6-week-old male SCID mice (MGH) as described previously (25) at 10-fold fewer cells than normally injected. For PC3M-LN4, osmotic pumps were implanted on the day of injection and changed once at two weeks. For liposarcoma clone 4, osmotic pumps were implanted on the day of injection and changed once a month for 3 months. For GFP-labeled metastasis studies, LLC-GFP or B16F10-GFP was injected subcutaneously into 6-week-old male C57BL/ 6 mice (The Jackson Laboratory) at $1 \times 10^{6}$ cells; tumors were resected once they had reached a size of $2-4 \mathrm{~cm}^{3}$, and osmotic pumps were implanted on the day of resection and changed every two weeks. B16F10-GFP was injected in 6-week-old male C57BL/ 6 (The Jackson Laboratory) intravenously at $2.5 \times 10^{5}$ cells, and osmotic pumps were changed every 2 weeks.

Parabiosis. Parabiosis was performed and cross-circulation assessed in a subset of animals ( $n=3$ couples) from each study condition as we previously described (54). Organs were harvested 3 hours after injection from each animal for densitometric analysis to verify dye cross-circulation. For tumor and metastasis experiments, LLC tumors were resected 4 weeks after injection. For adoptive transfer of blood cells, whole blood was taken from the lowEET recipient parabiont (Tie2-sEH-Tr) and inoculated retro-orbitally (300 $\mu l)$ into non-parabiosis high-EET (Tie2-CYP2C8-Tr) and WT mice. For in vivo VEGF depletion, Ad-null and Ad-sFlt were administered systemically 5 days prior to tumor injection and 2 days after tumor injection: Ad-null, $1 \times 10^{9} \mathrm{U}$; Ad-sFlt, $1 \times 10^{9} \mathrm{U}$. Plasma sFlt levels were measured by ELISA (R\&D Systems). Quantification of ECs in the primary LLC tumors was performed by FACS following enzymatic digestion as described previously (59).

Flow cytometry analysis. For flow cytometry on corneal tumors, whole eyes were removed and single-cell suspensions were prepared by enzymatic digestions as described previously (59) and incubated with Liberase. The percentage of ECs (CD-31-positive and CD45-negative) was determined using PE- and APC-conjugated antibodies (Roche). Digested tissue was filtered through a cell strainer and resuspended in FACS Buffer (PBS, 1\% BSA, $5 \mathrm{mM}$ EDTA/0.05\% sodium azide). Flow cytometry was performed using FACSCalibur and CellQuest software (BD Biosciences). For LLC-GFP, only the population of FL1 ${ }^{\text {hi }}$ cells (GFP-expressing cells) was collected for analysis. Data were collected using CellQuest software (BD Biosciences) and analyzed using WinMDI 2.8 software (http://facs.scripps.edu/software.html).

For macrophage depletion studies, macrophages were depleted by clodronate liposomes, and depletion was confirmed by flow cytometry as described previously (30).

Western blot analysis. We isolated tumor ECs and normal quiescent ECs as we previously described (52) and analyzed ECs, murine prostate tumor cells (TRAMP C1), and LLC tumor lysates from small (LLC $<1 \mathrm{~cm}^{3}$ ) and larger $\left(\mathrm{LLC}>5 \mathrm{~cm}^{3}\right.$ ), rapidly growing tumors for the expression of sEH, CYP2C, and CYP2J by Western blotting. Western blot analysis of tumors was performed as we described previously (32). Plasma from LLC tumor-bearingmice was purified using a heparin bead affinity technique and analyzed for TSP1 as we described (60).

Immunohistochemistry. Tumor samples were processed and immunohistochemical stainings performed as described previously (28). PECAM (CD31), MECA-32, and CD45 staining was performed as described (60). Sections were microwaved in $10 \mathrm{mM}$ sodium citrate ( $\mathrm{pH}$ 6.0) for Ki67, TSP1 (Lab Vision), and VEGF (Lab Vision). Sections were microwaved in EDTA (Zymed, Invitrogen) for VEGFR2 (Cell Signaling Technology). For sEH (Santa Cruz Biotechnology Inc.), sections were heated in citrate in a pressure cooker at $120^{\circ} \mathrm{C}$ for 3 minutes. Ki67 (Vector Laboratories), sEH (Santa Cruz Biotechnology Inc.), GFP (Abcam), and VEGF (Thermo Scientific) were incubated at $4^{\circ} \mathrm{C}$ overnight. Histological sections of tumors were analyzed for vessel density as described (32). $\beta$-Galactosidase staining was performed as described (50). Immunostaining and localization of GFPlabeled tumors were analyzed using a confocal Leica SP2 microscope.

Angiogenesis assay. All animal studies were reviewed and approved by the Animal Care and Use Committee of Children's Hospital, Boston. For corneal tumor angiogenesis, LLC, LLC-GFP, and B16F10 were injected into the cornea, and angiogenic response was recorded as we described previously (28). 14,15-EET or vehicle osmotic pumps were implanted on the day of injection and changed once at 2 weeks after injection. Histological sections of tumors were analyzed for microvessel density was as described (32). EC migration assays were performed as we described (61). ECs were pretreated with $t$ AUCB, TUPS, 14,15-EEZE, or vehicle for 30 minutes in suspension. VEGF was measured by ELISA as we previously described (32). TSP1 was measured by ELISA. Tumor cells (human prostate PC3M-LN4 cells), ECs (human microvascular ECs [HMVECs]), or fibroblasts (human) were plated at 20,000 cells per well and 24 hours later were treated with 14,15 -EET $(1 \mu \mathrm{M})$ or vehicle every 8 hours for 24 hours. Medium was collected 24 hours after drug challenge. Quantitative RT-PCR was performed with the Qantitect SYBR Green RT-PCR kit (QIAGEN) using the ABI 
7300 real-time PCR system (Applied Biosystems); cyclophilin controlled for complementary DNA content. The primers used were mouse VEGFR2 (forward: 5'-GCCCTGCCTGGTCTCACTAC-3', reverse: 5'-CAAAGCATTGCCCATTCGAT-3'), mouse VEGFR1 (forward: 5'-TGGCTCTACGACCTTAGACTG-3', reverse: 5'-CAGGTTTGACTTGTCTGAGGTT-3'), mouse cyclophilin (forward: 5'-CAGACGCCACTGTCGCTTT-3', reverse: 5'TGTCTTTGGAACTTTGTCTGCAA-3').

LC-MS-MS analysis. Aortic ECs were isolated from WT, Tie2-CYP2J2-Tr, and Tie2-CYP2C8-Tr mice as previously described (62). Confluent $10-\mathrm{cm}$ plates were treated with $10 \mu \mathrm{M}$ A23187 for 15 minutes. Media were collected and assayed for 14,15-EET by LC/MS/MS. Kidney ECs isolated from WT and Tie2-sEH-Tr mice were incubated in serum-free medium for 4 hours. Media were collected and assayed for 14,15-EET by LC/MS/MS. Eicosanoid levels in plasma, tumors (TRAMP and T241 fibrosarcoma), and normal prostate tissue were analyzed by LC/MS/MS as previously described (19).

Statistics. Student's 2-sided unpaired $t$ test was used to analyze the difference between the two groups. $P$ value less than 0.05 were considered statistically significant. Data are presented as mean \pm SEM.

\section{Acknowledgments}

We thank and gratefully acknowledge Kristin Johnson for photography and preparation of the figures; J. Alyce Bradbury, Joan P. Graves, Laura M. DeGraff, Ricky Sanchez, Lyndsay Leach, and Tony Walshe for excellent technical assistance; and Sung Hee Hwang for sEH inhibitors and eicosanoid profiling assays. We thank Isaiah J. Fidler for PC3M-LN4 cells. This work was supported by National
Cancer Institute grant R01CA148633-01A1 (to D. Panigrahy); the Stop and Shop Pediatric Brain Tumor Fund and the C.J. Buckley Pediatric Brain Tumor Fund (to M.W. Kieran); Division of Intramural Research of the NIH, National Institute of Environmental Health Sciences grants Z01 ES025034 and Z01 ES050167 (to D.C. Zeldin and K.B. Tomer); NIH grant R01 GM088199 (to C.R. Lee); the Robert A. Welch Foundation (to J.R. Falck); NIEHS Superfund Basic Research Program grants NIH P42 ES004699, NIH R01 ES002710, and NIH R01 ES013933 (to B.D. Hammock); and NIH grant CA045548 (to D.E. Ingber). B.D. Hammock is a George and Judy Marcus Senior Fellow of the American Asthma Foundation.

Received for publication March 22, 2011, and accepted in revised form October 12, 2011.

Address correspondence to: Dipak Panigrahy, 300 Longwood Avenue, Boston, Massachusetts 02115, USA. Phone: 617.919.2198; Fax: 617.730.0231; E-mail: dipak.panigrahy@childrens.harvard.edu. Or to: Sui Huang, 401 Terry Avenue North, Seattle, Washington 98109, USA. Phone: 206.354.3569; Fax: 206.732.1299; E-mail: sui.huang@ systemsbiology.org. Or to: Mark W. Kieran, 450 Brookline Avenue, SW331, Boston, Massachusetts 02115, USA. Phone: 617.632.4907; Fax: 617.632.4897; E-mail: mark_kieran@dfci.harvard.edu. Or to: Darryl C. Zeldin, 111 TW Alexander Drive, Building 101, A214 Research Triangle Park, North Carolina 27709, USA. Phone: 919.541.1169; Fax: 919.541.4214; E-mail: zeldin@niehs.nih.gov.
1. Gronert K. Lipid autacoids in inflammation and injury responses: a matter of privilege. Mol Interv. 2008;8(1):28-35.

2. Campbell WB, Falck JR. Arachidonic acid metabolites as endothelium-derived hyperpolarizing factors. Hypertension. 2007;49(3):590-596

3. Fleming I. Vascular cytochrome p450 enzymes: physiology and pathophysiology. Trends Cardiovasc Med. 2008;18(1):20-25.

4. Imig JD, Hammock BD. Soluble epoxide hydrolase as a therapeutic target for cardiovascular diseases. Nat Rev Drug Discov. 2009;8(10):794-805.

5. Shen HC. Soluble epoxide hydrolase inhibitors: a patent review. Expert Opin Ther Pat. 2010;20(7):941-956.

6. Munzenmaier DH, Harder DR. Cerebral microvascular endothelial cell tube formation: role of astrocytic epoxyeicosatrienoic acid release. Am J Physiol Heart Circ Physiol. 2000;278(4):H1163-H1167.

7. Pozzi A, et al. Characterization of 5,6- and 8,9epoxyeicosatrienoic acids (5,6- and 8,9-EET) as potent in vivo angiogenic lipids. J Biol Chem. 2005; 280(29):27138-27146

8. Wang Y, et al. Arachidonic acid epoxygenase metabolites stimulate endothelial cell growth and angiogenesis via mitogen-activated protein kinase and phosphatidylinositol 3-kinase/Akt signaling pathways. J Pharmacol Exp Ther. 2005;314(2):522-532.

9. Panigrahy D, Kaipainen A, Greene ER, Huang S. Cytochrome P450-derived eicosanoids: the neglected pathway in cancer. Cancer Metastasis Rev. 2010; 29(4):723-735.

10. Jiang JG, et al. Cytochrome P450 2J2 promotes the neoplastic phenotype of carcinoma cells and is up-regulated in human tumors. Cancer Res. 2005; 65(11):4707-4715.

11. Jiang JG, et al. Cytochrome p450 epoxygenase promotes human cancer metastasis. Cancer Res. 2007; 67(14):6665-6674.

12. Jernstrom H, Bageman E, Rose C, Jonsson PE, Ingvar C. CYP2C8 and CYP2C9 polymorphisms in relation to tumour characteristics and early breast cancer related events among 652 breast cancer patients. BrJ Cancer. 2009;101(11):1817-1823.
13. Zagorac D, Jakovcevic D, Gebremedhin D, Harder DR. Antiangiogenic effect of inhibitors of cytochrome P450 on rats with glioblastoma multiforme. J Cereb Blood Flow Metab. 2008;28(8):1431-1439.

14. Node K, et al. Anti-inflammatory properties of cytochrome P450 epoxygenase-derived eicosanoids. Science. 1999;285(5431):1276-1279.

15. Lee CR, et al. Endothelial expression of human cytochrome P450 epoxygenases lowers blood pressure and attenuates hypertension-induced renal injury in mice. FASEB J. 2010;24(10):3770-3781.

16. Sinal CJ, Miyata M, Tohkin M, Nagata K, Bend JR, Gonzalez FJ. Targeted disruption of soluble epoxide hydrolase reveals a role in blood pressure regulation. J Biol Chem. 2000;275(51):40504-40510.

17. Seubert JM, et al. Role of soluble epoxide hydrolase in postischemic recovery of heart contractile function. Circ Res. 2006;99(4):442-450.

18. Edin ML, et al. Endothelial expression of human cytochrome P450 epoxygenase CYPC8 increases susceptibility to ischemia-reperfusion injury in the isolated mouse heart. FASEB J. 2011;25(10):3436-3447.

19. Newman JW, Watanabe T, Hammock BD. The simultaneous quantification of cytochrome $\mathrm{P} 450$ dependent linoleate and arachidonate metabolites in urine by HPLC-MS/MS.J Lipid Res. 2002;43(9):1563-1578.

20. Pettaway CA, et al. Selection of highly metastatic variants of different human prostatic carcinomas using orthotopic implantation in nude mice. Clin Cancer Res. 1996;2(9):1627-1636.

21. Jeet V, Russell PJ, Khatri A. Modeling prostate cancer: a perspective on transgenic mouse models. Cancer Metastasis Rev. 2010;29(1):123-142.

22. Almog N. Molecular mechanisms underlying tumor dormancy. Cancer Lett. 2010;294(2):139-146.

23. Goss PE, Chambers AF. Does tumour dormancy offer a therapeutic target? Nat Rev Cancer. 2010; 10(12):871-877.

24. Naumov GN, et al. A model of human tumor dormancy: an angiogenic switch from the nonangiogenic phenotype. J Natl Cancer Inst. 2006;98(5):316-325.

25. Holmgren L, O'Reilly MS, Folkman J. Dormancy of micrometastases: balanced proliferation and apop- tosis in the presence of angiogenesis suppression. Nat Med. 1995;1(2):149-153.

26. Almog N, et al. Prolonged dormancy of human liposarcoma is associated with impaired tumor angiogenesis. FASEB J. 2006;20(7):947-949.

27. Naumov GN, MacDonald IC, Chambers AF, Groom AC. Solitary cancer cells as a possible source of tumour dormancy? Semin Cancer Biol. 2001;11(4):271-276.

28. Kaipainen A, et al. PPARalpha deficiency in inflammatory cells suppresses tumor growth. PLoS One. 2007;2(2):e260.

29. De Palma M, et al. Tumor-targeted interferon-alpha delivery by Tie2-expressing monocytes inhibits tumor growth and metastasis. Cancer Cell. 2008; 14(4):299-311.

30. Zeisberger SM, Odermatt B, Marty C, ZehnderFjällman AH, Ballmer-Hofer K, Schwendener RA. Clodronate-liposome-mediated depletion of tumour-associated macrophages: a new and highly effective antiangiogenic therapy approach. BrJ Cancer. 2006;95(3):272-281.

31. O'Reilly MS, et al. Angiostatin: a novel angiogenesis inhibitor that mediates the suppression of metastases by a Lewis lung carcinoma. Cell. 1994; 79(2):315-328.

32. Panigrahy D, et al. PPARgamma ligands inhibit primary tumor growth and metastasis by inhibiting angiogenesis. J Clin Invest. 2002;110(7):923-932.

33. Parhar RS, Lala PK. Amelioration of B16F10 melanoma lung metastasis in mice by a combination therapy with indomethacin and interleukin 2. JExp Med. 1987;165(1):14-28.

34. Barbera-Guillem E, Smith I, Weiss L. Cancer-cell traffic in the liver. I. Growth kinetics of cancer cells after portal-vein delivery. Int J Cancer. 1992;52(6):974-977.

35. Price JE, Tarin D, Fidler IJ. Influence of organ microenvironment on pigmentation of a metastatic murine melanoma. Cancer Res. 1988;48(8):2258-2264.

36. Hwang SH, Tsai HJ, Liu JY, Morisseau C, Hammock BD. Orally bioavailable potent soluble epoxide hydrolase inhibitors. J Med Chem. 2007;50(16):3825-3840.

37. Gauthier KM, et al. 14,15-Epoxyeicosa-5(Z)-enoic acid: a selective epoxyeicosatrienoic acid antagonist 
that inhibits endothelium-dependent hyperpolarization and relaxation in coronary arteries. Circ Res. 2002;90(9):1028-1036.

38. Gauthier KM, Jagadeesh SG, Falck JR, Campbell WB. 14,15-epoxyeicosa-5(Z)-enoic-mSI: a 14,15and 5,6-EET antagonist in bovine coronary arteries. Hypertension. 2003;42(4):555-561.

39. Deng Y, et al. Endothelial CYP epoxygenase overexpression and soluble epoxide hydrolase disruption attenuate acute vascular inflammatory responses in mice. FASEB J. 2011;25(2):703-713.

40. Iliff JJ, Wang R, Zeldin DC, Alkayed NJ. Epoxyeicosanoids as mediators of neurogenic vasodilation in cerebral vessels. Am J Physiol Heart Circ Physiol. 2009;296(5):H1352-H1363.

41. Motoki A, et al. Soluble epoxide hydrolase inhibition and gene deletion are protective against myocardial ischemia-reperfusion injury in vivo. Am J Physiol Heart Circ Physiol. 2008;295(5):H2128-H2134.

42. Webler AC, et al. Epoxyeicosatrienoic acids are part of the VEGF-activated signaling cascade leading to angiogenesis. Am J Physiol Cell Physiol. 2008; 295(5):C1292-C1301.

43. Gross GJ, et al. Effects of the selective EET antagonist, 14,15-EEZE, on cardioprotection produced by exogenous or endogenous EETs in the canine heart. Am J Physiol Heart Circ Physiol. 2008; 294(6):H2838-H2844.

44. Hanahan D, Folkman J. Patterns and emerging mechanisms of the angiogenic switch during tumorigenesis. Cell. 1996;86(3):353-364.

45. Hicklin DJ, Ellis LM. Role of the vascular endothelial growth factor pathway in tumor growth and angio- genesis. J Clin Oncol. 2005;23(5):1011-1027.

46. Shibuya M, Claesson-Welsh L. Signal transduction by VEGF receptors in regulation of angiogenesis and lymphangiogenesis. Exp Cell Res. 2006;312(5):549-560.

47. Kuo CJ, et al. Comparative evaluation of the antitumor activity of antiangiogenic proteins delivered by gene transfer. Proc Natl Acad Sci U S A. 2001; 98(8):4605-4610.

48. Streit M, et al. Overexpression of thrombospondin-1 decreases angiogenesis and inhibits the growth of human cutaneous squamous cell carcinomas. Am J Pathol. 1999;155(2):441-452.

49. Fukumura D, et al. Tumor induction of VEGF promoter activity in stromal cells. Cell. 1998; 94(6):715-725.

50. Maharaj AS, Saint-Geniez M, Maldonado AE, D'Amore PA. Vascular endothelial growth factor localization in the adult. Am J Pathol. 2006; 168(2):639-648.

51. Enayetallah AE, French RA, Grant DF. Distribution of soluble epoxide hydrolase, cytochrome P450 $2 \mathrm{C} 8,2 \mathrm{C} 9$ and $2 \mathrm{~J} 2$ in human malignant neoplasms. J Mol Histol. 2006;37(3-4):133-141.

52. Dudley AC, et al. Calcification of multipotent prostate tumor endothelium. Cancer Cell. 2008;14(3):201-211.

53. Nakamura T, Kuwai T, Kim JS, Fan D, Kim SJ, Fidler IJ. Stromal metalloproteinase-9 is essential to angiogenesis and progressive growth of orthotopic human pancreatic cancer in parabiont nude mice. Neoplasia. 2007;9(11):979-986.

54. Pietramaggiori G, Scherer SS, Alperovich M, Chen B, Orgill DP, Wagers AJ. Improved cutaneous healing in diabetic mice exposed to healthy peripheral circu- lation. J Invest Dermatol. 2009;129(9):2265-2274.

55. Folkman J. Angiogenesis. In: Braunwald E, Fauci AS, Kasper DL, Hauser SL, Longo DL, Jameson JL, eds. Harrison's Textbook of Internal Medicine. New York, New York, USA: McGraw-Hill; 2001:517-530.

56. Harach HR, Fransilla KO, Wasenius VM. Occult papillary carcinoma of the thyroid. A "normal" finding in Finland. A systemic autopsy study. Cancer. 1985; 56(3):531-538.

57. Barkan D, Green JE, Chambers AF. Extracellular matrix: a gatekeeper in the transition from dormancy to metastatic growth. Eur J Cancer. 2010; 46(7):1181-1188.

58. Schlaeger TM, et al. Uniform vascular-endothelialcell-specific gene expression in both embryonic and adult transgenic mice. Proc Natl Acad Sci US A. 1997;94(7):3058-3063.

59. Adini A, Fainaru O, Udagawa T, Connor KM, Folkman J, D'Amato RJ. Matrigel cytometry: a novel method for quantifying angiogenesis in vivo. J Immunol Methods. 2009;342(1-2):78-81.

60. Panigrahy D, et al. PPARalpha agonist fenofibrate suppresses tumor growth through direct and indirect angiogenesis inhibition. Proc Natl Acad SciUS A. 2008;105(3):985-990.

61. Short SM, Derrien A, Narsimhan RP, Lawler J, Ingber DE, Zetter BR. Inhibition of endothelial cell migration by thrombospondin- 1 type- 1 repeats is mediated by beta 1 integrins. J Cell Biol. 2005; 168(4):643-653.

62. Lim YC, et al. Heterogeneity of endothelial cells from different organ sites in T-cell subset recruitment. Am J Pathol. 2003;162(5):1591-1601. 